ARTICLE

\title{
Nlrp12 mutation causes C57BL/6J strain-specific defect in neutrophil recruitment
}

Tyler K. Ulland ${ }^{1,2, \star}$, Nidhi Jain ${ }^{1,3, \star}$, Emma E. Hornick ${ }^{1,4}$, Eric I. Elliott1,2,3,5, Gwendolyn M. Clay 2,5 , Jeffrey J. Sadler ${ }^{1}$, Kathleen A.M. Mills ${ }^{1}$, Ann M. Janowski ${ }^{1,4}$, A. Paige Davis Volk ${ }^{1,6}$, Kai Wang ${ }^{7}$, Kevin L. Legge ${ }^{4,5,8}$, Lokesh Gakhar 9,10 , Mohammed Bourdi ${ }^{11}$, Polly J. Ferguson ${ }^{6}$, Mary E. Wilson $2,4,5,12,13,14$, Suzanne L. Cassel ${ }^{1,3,4,12, \star, \star \star}$

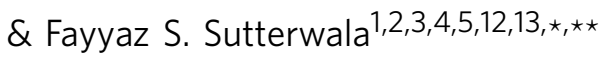

The inbred mouse strain C57BL/6J is widely used in models of immunological and infectious diseases. Here we show that C57BL/6J mice have a defect in neutrophil recruitment to a range of inflammatory stimuli compared with the related $\mathrm{C} 57 \mathrm{BL} / 6 \mathrm{~N}$ substrain. This immune perturbation is associated with a missense mutation in N/rp12 in C57BL/6J mice. Both C57BL/6J and NLRP12-deficient mice have increased susceptibility to bacterial infection that correlates with defective neutrophil migration. C57BL/6J and NLRP12-deficient macrophages have impaired CXCL1 production and the neutrophil defect observed in C57BL/6J and NLRP12-deficient mice is rescued by restoration of macrophage NLRP12. These results demonstrate that C57BL/6J mice have a functional defect in NLRP12 and that macrophages require NLRP12 expression for effective recruitment of neutrophils to inflammatory sites.

\footnotetext{
${ }^{1}$ Inflammation Program, University of lowa Carver College of Medicine, lowa City, lowa 52242, USA. ${ }^{2}$ Interdisciplinary Program in Molecular and Cellular Biology, University of lowa Carver College of Medicine, lowa City, lowa 52242, USA. ${ }^{3}$ Department of Medicine, Cedars-Sinai Medical Center, Los Angeles, California 90048, USA. ${ }^{4}$ Interdisciplinary Program in Immunology, University of lowa Carver College of Medicine, lowa City, lowa 52242, USA. ${ }^{5}$ Medical Scientist Training Program, University of lowa Carver College of Medicine, lowa City, lowa 52242, USA. ${ }^{6}$ Department of Pediatrics, University of lowa Carver College of Medicine, lowa City, lowa 52242, USA. ${ }^{7}$ Department of Biostatistics, University of lowa College of Public Health, lowa City, lowa 52242, USA. 8 Department of Pathology, University of lowa Carver College of Medicine, lowa City, lowa 52242, USA. ${ }^{9}$ Department of Biochemistry, University of lowa Carver College of Medicine, lowa City, lowa 52242, USA. ${ }^{10}$ Protein Crystallography Facility, University of lowa Carver College of Medicine, lowa City, lowa 52242, USA. ${ }^{11}$ Molecular and Cellular Toxicology Section, Laboratory of Molecular Immunology, National Heart, Lung and Blood Institute, National Institutes of Health, Bethesda, Maryland 20892, USA. ${ }^{12}$ Department of Internal Medicine, University of lowa Carver College of Medicine, lowa City, lowa 52242, USA. ${ }^{13}$ Veterans Affairs Medical Center, lowa City, lowa 52246, USA. ${ }^{14}$ Department of Microbiology, University of lowa Carver College of Medicine, lowa City, lowa 52242, USA. * These authors contributed equally to this work. ${ }^{\star \star}$ These authors jointly supervised this work. Correspondence and requests for materials should be addressed to F.S.S. (email: fayyaz.sutterwala@cshs.org).
} 
T he genetic background of a transgenic or knockout mouse has an important, but often overlooked, role in its phenotype. The founder C57BL/6J substrain originated in the 1920s and has been maintained at the Jackson Laboratories since 1948. A colony was established at the National Institutes of Health in 1951 and designated as the C57BL/6N substrain. Over the intervening 65 years there has been substantial genetic drift. Comparison of the genome between these two substrains revealed 40 single nucleotide polymorphisms (SNPs) and 30 indels ${ }^{1-3}$. This genetic difference is relevant because large projects have utilized different substrains for their work. The International Mouse Phenotyping Consortium, for example, uses C57BL/6N embryonic stem cells for the generation of knockout mice. By contrast, the Mouse Genome Sequencing Consortium has relied on the C57BL/6J substrain. In addition, many commercially available knockout mice are on a mixed background with varying genetic contributions from both C57BL/6J and C57BL/6N substrains. Both substrains are widely used in biomedical research and hence it is critical to understand the phenotypic differences, their genetic causes, and in particular how these differences affect the outcomes of immunological studies using these mice.

The nucleotide-binding domain and leucine-rich repeat containing receptor (NLR) family of proteins is a group of intracellular receptors that are important for sensing both pathogen-associated and danger-associated molecular patterns ${ }^{4}$. NLRP12 was initially identified as a member of the NLR family reported to attenuate inflammatory responses ${ }^{5-7}$. NLRP12 has also been shown to inhibit colonic inflammation and tumorigenesis in mouse models through negative regulation of nuclear factor kappa-light-chain $(\mathrm{NF}-\kappa \mathrm{B})^{8,9}$. The role for NLRP12 in controlling bacterial infections is less clear. Vladimer et al. ${ }^{10}$ suggest that NLRP12 serves a pro-inflammatory role during infection with a modified Yersinia pestis strain through its ability to form a caspase-1 activating inflammasome that regulates interleukin (IL)-1 $\beta$ and IL-18 secretion. In marked contrast, Zaki et al. ${ }^{11}$ found an anti-inflammatory role for NLRP12 showing that NLRP12-deficient mice were resistant to infection with Salmonella typhimurium due to increased $\mathrm{NF}-\kappa \mathrm{B}$ and extracellular signal-regulated kinases activation. In yet a separate study, Allen et al. ${ }^{12}$ identified no role for NLRP12 in the in vivo host response to Klebsiella pneumoniae and Mycobacterium tuberculosis. Given these markedly discrepant findings and postulated mechanisms of action of NLRP12 in bacterial infection, a closer examination is warranted.

In this study, we demonstrate that C57BL/6J mice have a defect in recruitment of neutrophils to inflammatory sites in vivo. This immune perturbation in C57BL/6J mice is associated with a missense mutation within the leucine-rich repeat domain of Nlrp12 resulting in an arginine to lysine change (R1034K). The failure of neutrophil migration in C57BL/6J mice was phenocopied in Nlrp12 $12^{-/}$mice. By comparing C57BL/6N, C57BL/6J and Nlrp12-/- mice we further demonstrate that the role of NLRP12 in the recruitment of neutrophils to the site of inflammation is critical for host defense against bacterial pathogens. Expression of C57BL/6N NLRP12 in macrophages is required to mediate appropriate neutrophil recruitment. Furthermore, there is a defect in macrophages from C57BL/6J and Nlrp12 ${ }^{-/-}$mice to produce the neutrophil chemotactic chemokine CXCL1 in response to inflammatory stimuli. Altogether, our findings demonstrate that NLRP12 controls neutrophil migration to inflammatory sites and that a missense mutation in Nlrp12 in C57BL/6J mice renders these mice with a functional NLRP12 deficit.

\section{Results}

Reduced neutrophil recruitment in C57BL/6J mice. Although differences in response to acetaminophen-induced hepatotoxicity and endotoxic shock have been noted in C57BL/6 substrains the genetic cause has not been determined ${ }^{2,13}$. We evaluated the ability of C57BL/6N and C57BL/6J mice to recruit neutrophils in response to inflammatory stimuli in vivo. C57BL/6N and C57BL/6J mice were challenged intranasally (i.n.) with lipopolysaccharide (LPS) or F. tularensis live vaccine strain (LVS) and the number of neutrophils in the bronchoalveolar lavage (BAL) quantified by flow cytometry. The total number of neutrophils present in the BAL of $\mathrm{C} 57 \mathrm{BL} / 6 \mathrm{~J}$ mice was diminished compared with that from C57BL/6N mice for both stimuli (Fig. 1a,b, Supplementary Fig. 1a-f). This C57BL/6 substrain difference was specific to the neutrophilic response, as numbers of $\mathrm{CD} 45.2^{+} \mathrm{Gr} 1^{-}$cells were not significantly different between C57BL/6N and C57BL/6J mice challenged i.n. with LPS (Supplementary Fig. 1g,h).

C57BL/6J mice also demonstrated increased mortality in response to i.n. challenge with $F$. tularensis LVS compared with C57BL/6N mice (Fig. 1c). Taken together, these data demonstrate that $\mathrm{C} 57 \mathrm{BL} / 6 \mathrm{~J}$ mice have diminished inflammatory responses compared with $\mathrm{C} 57 \mathrm{BL} / 6 \mathrm{~N}$ mice and this translates to increased susceptibility to bacterial infection in vivo.

C57BL/6J Nlrp12 mutation causes defective neutrophil recruitment. Commensal intestinal bacteria can have profound effects on the host immune system. Ivanov et al. ${ }^{14}$ demonstrated that differences in segmented filamentous bacterium (SFB) colonization between C57BL/6 mice from Jackson Laboratories and Taconic Farms influenced Th17 cell differentiation. To assess the possibility that the differences in neutrophilic responses observed between $\mathrm{C} 57 \mathrm{BL} / 6 \mathrm{~N}$ and $\mathrm{C} 57 \mathrm{BL} / 6 \mathrm{~J}$ mice was due to differences in intestinal microbiota, C57BL/6N and C57BL/6J mice were co-housed for 4 weeks before i.n. LPS challenge. A significant decrease in neutrophil recruitment in the BAL was still observed in C57BL/6J mice that had been co-housed with C57BL/6N mice (Fig. 1d) suggesting a predominant genetic rather than environmental cause for this phenotypic difference. We did observe that $\mathrm{C} 57 \mathrm{BL} / 6 \mathrm{~N}$ mice that had been co-housed with C57BL/6J mice had decreased neutrophilic responses compared with separately housed C57BL/6N mice (Fig. 1d) implying that there may be an additional role for microbiota in neutrophil recruitment.

To identify the genetic cause of the neutrophil defect observed in $\mathrm{C} 57 \mathrm{BL} / 6 \mathrm{~J}$ mice we intercrossed $\mathrm{C} 57 \mathrm{BL} / 6 \mathrm{~N}$ and $\mathrm{C} 57 \mathrm{BL} / 6 \mathrm{~J}$ mice (Supplementary Fig. 2a $)^{15}$. We found $\mathrm{F}_{1}$ offspring had neutrophil recruitment similar to $\mathrm{C} 57 \mathrm{BL} / 6 \mathrm{~J}$ mice in response to i.n. LPS challenge, suggesting the C57BL/6J trait was dominant (Fig. 1e). Others have compared the genome between $\mathrm{C} 57 \mathrm{BL} / 6 \mathrm{~N}$ and C57BL/6J substrains and over 40 SNPs and 30 indels have been identified that differ between the substrains ${ }^{1-3,16}$. To determine which genetic loci contribute to the reduced neutrophil recruitment observed in C57BL/6J mice we took a candidate gene approach. $F_{1}$ mice were intercrossed and $73 \mathrm{~F}_{2}$ offspring were obtained and analysed by sequencing for the presence of the previously identified SNPs and indels. C57BL/6J mice have been reported to carry a missense polymorphism in chromosome 7 at position 3,222,537 ( $\mathrm{G}$ to A) leading to the substitution of a lysine for an arginine in the $\mathrm{C}$-terminal leucinerich repeat domain of NLRP12 (ref. 1), which we confirmed (Fig. If and Supplementary Fig. 2b). We genotyped the $F_{2}$ mice for this mutation and found the presence of this missense polymorphism in Nlrp12 in C57BL/6J mice correlated with defective neutrophil recruitment (Fig. 1e). Analysis of two other 
a

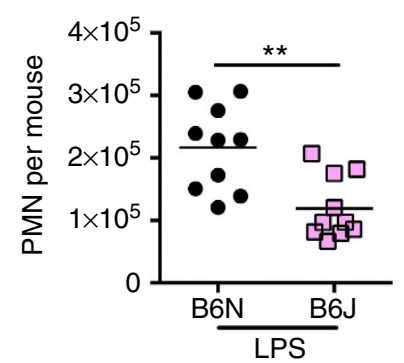

d

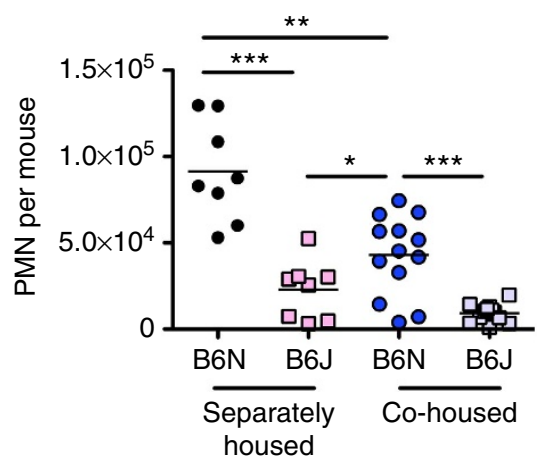

b

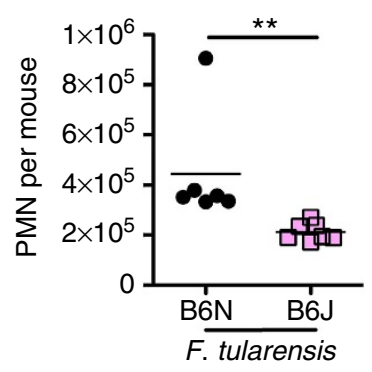

C

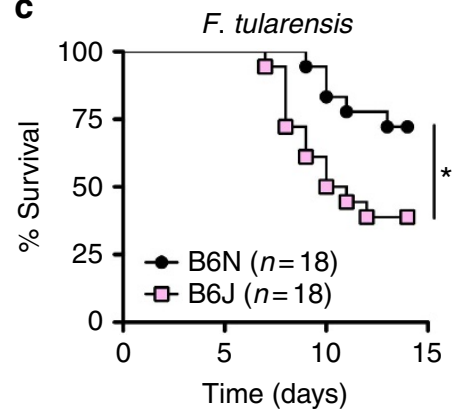

e

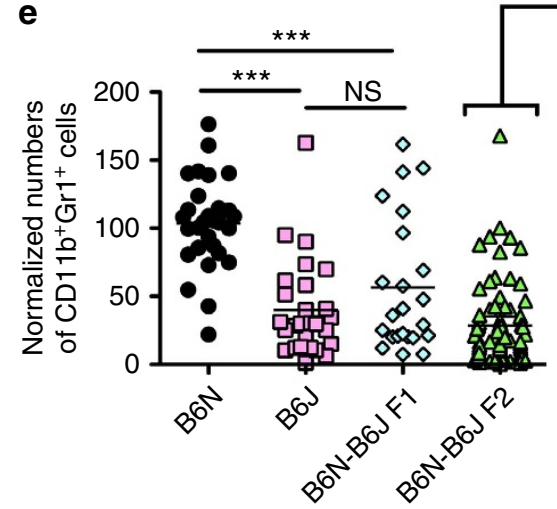

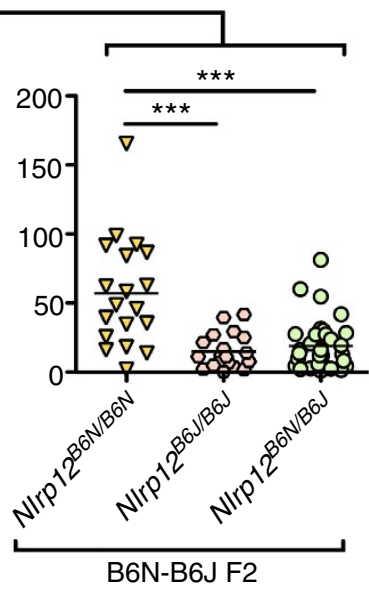

f
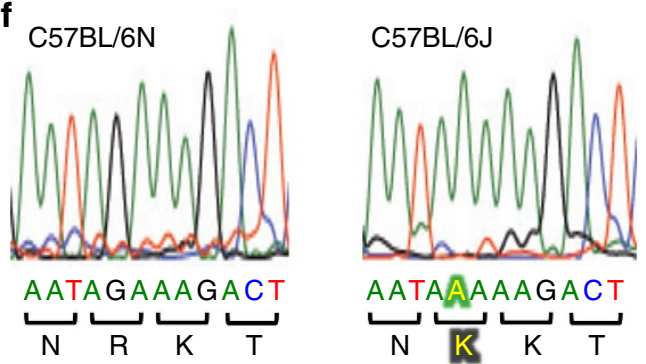

Figure 1 | NIrp12 mutation in C57BL/6J mice results in defective neutrophil recruitment. (a,b) C57BL/6N (B6N) and C57BL/6J (B6J) mice were challenged i.n. with LPS ( $0.5 \mathrm{mg} \mathrm{kg}^{-1}$ of body weight) ( $n=10$ per group) (a), or $5 \times 10^{3}$ colony-forming unit (CFU) of $F$. tularensis LVS ( $n=6$ per group) (b). Six hours (a) or $72 \mathrm{~h}(\mathbf{b})$ post challenge BAL were performed and the number of $\mathrm{Gr}^{+}$neutrophils quantified by flow cytometry. (c) B6N and B6J mice were challenged i.n. with $5 \times 10^{3}$ CFU of $F$. tularensis LVS and survival monitored. (d) B6N and B6J mice were either housed separately or co-housed at a 1:1 ratio for 4 weeks before i.n. challenge with LPS. Neutrophil influx (CD45.2 $\left.{ }^{+} \mathrm{CD} 11 \mathrm{~b}{ }^{+} \mathrm{Gr}^{+}\right)$into the BAL was determined $6 \mathrm{~h}$ post challenge by flow cytometry ( $n=8$, separately housed group; $n \geq 12$, co-housed group). (e) B6N, B6J, B6N-B6J F1 and B6N-B6J F2 mice were challenged i.n. with LPS. Six hours post challenge BAL were performed and the number of neutrophils (CD45.2 ${ }^{+} \mathrm{CD}_{11 \mathrm{~b}}{ }^{+} \mathrm{Gr} 1^{+}$) quantified by flow cytometry. The Nlrp12 allele was sequenced for all B6N-B6J F2 mice and cohorts stratified based on their N/rp12 genotype (N/rp12 ${ }^{B 6 N / B 6 N}$, N/rp12 ${ }^{B 6 N / B 6 J}$ or N/rp12 $\left.2^{B 6 J / B 6 J}\right)(n=29, B 6 N$; $n=27, \mathrm{~B} 6 \mathrm{~J} ; n=23, \mathrm{F1} ; n=73, \mathrm{F2}$ ). (f) Nucleotide sequence profile of the N/rp12 allele in B6N and B6J mice. The highlighted text indicates the presence of a $\mathrm{G}$ to $\mathrm{A}$ missense polymorphism in $\mathrm{C} 57 \mathrm{BL} / 6 \mathrm{~J}$ mice leading to the change of an arginine for lysine at position 1034 of $\mathrm{NLRP12}$. ${ }^{\star} P<0.05$, ${ }^{\star \star} P<0.01$,

${ }^{\star \star \star} P<0.005, \mathrm{NS}$, not significant by Mann-Whitney U-test $(\mathbf{a}, \mathbf{b}, \mathbf{d}, \mathbf{e})$ or log-rank test $(\mathbf{c})$.

genes did not reveal any correlation to diminished neutrophil recruitment in C57BL/6J mice (Supplementary Fig. 2c,d). Consistent with the phenotype of $\mathrm{F}_{1}$ mice, $\mathrm{F}_{2}$ mice that were heterozygous at the Nlrp12 locus had neutrophil recruitment similar to $\mathrm{C} 57 \mathrm{BL} / 6 \mathrm{~J}$ mice in response to i.n. LPS challenge, further suggesting the dominance of the C57BL/6J trait (Fig. 1e).

Haematopoietic NLRP12 controls neutrophil recruitment. To specifically determine if NLRP12 was required for effective neutrophil recruitment to inflammatory stimuli, we utilized mice in which Nlrp12 had been genetically ablated. Similar to C57BL/6J mice, Nlrp12-/- mice challenged i.n. with LPS displayed significantly diminished neutrophilic influx into the BAL fluid from lungs, as compared with wild-type (WT) C57BL/6N mice (Fig. 2a). Nlrp12-/- mice backcrossed onto a BALB/cJ background also had a neutrophil recruitment defect in response to intranasal LPS challenge (Fig. 2b). Taken together, these data demonstrate a defect in neutrophil migration in response to LPS in $N$ lrp12 1 - mice.

In addition to neutrophilic infiltration, vascular leakage has an important role in the pathology of LPS-induced acute lung injury. Therefore, we examined the role of NLRP12 in LPS-induced increase in microvascular permeability. In WT (C57BL/6N) mice, LPS inhalation caused an increase in Evans blue leakage into the lung (Fig. 2c). In contrast, Nlrp12 $2^{-/-}$mice were protected from 

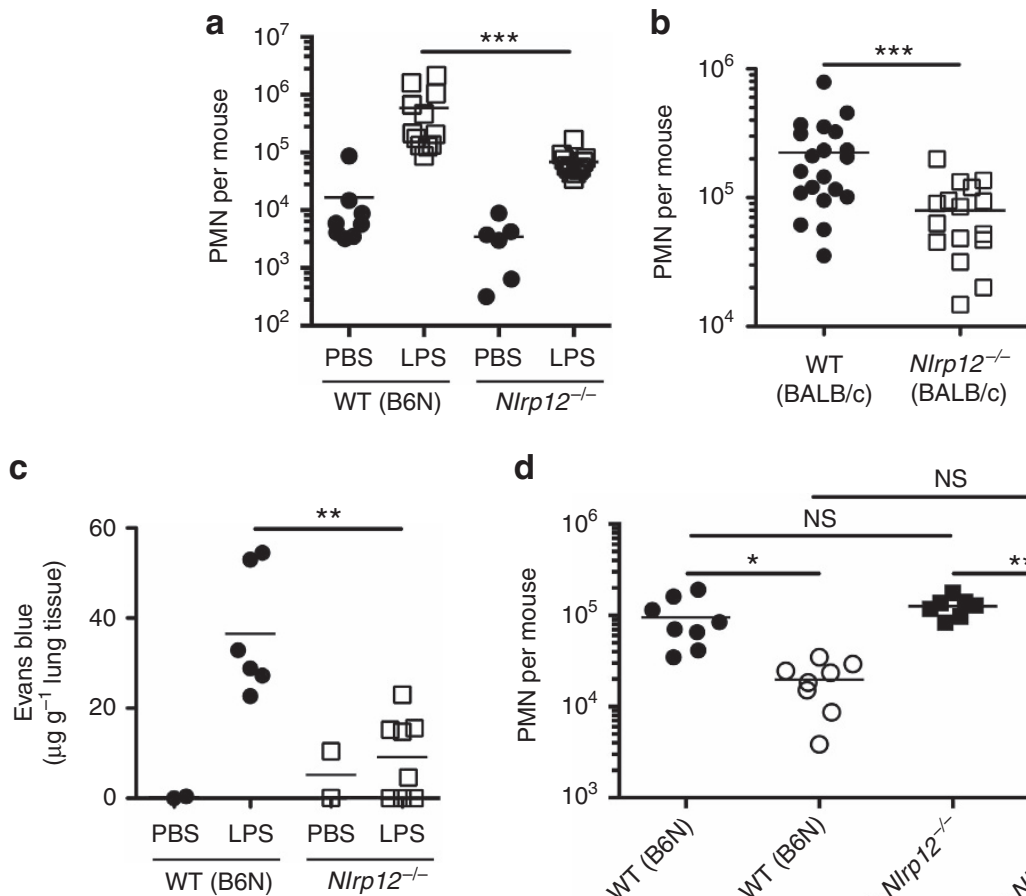

d

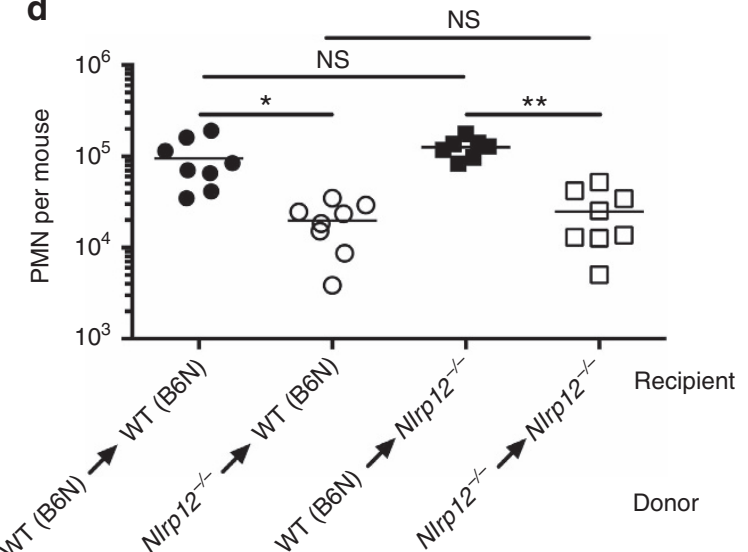

Figure 2 | Haematopoietic NIrp12 expression is required for effective neutrophil recruitment. (a) WT (B6N) or N/rp12-/- mice were challenged i.n. with either PBS or LPS $\left(0.5 \mathrm{mg} \mathrm{kg}^{-1}\right.$ of body weight). Six hours post challenge BAL were performed and the number of $\mathrm{Gr} 1^{+}$cells quantified by flow cytometry ( $n \geq 6$, PBS-treated group; $n=12$, LPS-treated group). (b) WT (BALB/cJ) or N/rp12-/- mice on a BALB/cJ background were challenged i.n. with LPS ( $0.0125 \mathrm{mg} \mathrm{kg}^{-1}$ of body weight). Six hours post challenge BAL were performed and the number of $\mathrm{Gr} 1^{+}$cells quantified by flow cytometry ( $n \geq 17$ per group). (c) WT (B6N) or Nlrp12-/- mice were challenged i.n. with either PBS or LPS ( $0.5 \mathrm{mg} \mathrm{kg}^{-1}$ of body weight). In all, $5.5 \mathrm{~h}$ post challenge mice were injected i.v. with Evans blue dye $\left(20 \mathrm{mg} \mathrm{kg}^{-1}\right)$. At $6 \mathrm{~h}$ post LPS challenge, the amount of extravasated Evans blue dye into the lung was calculated ( $n=2$, PBS-treated group; $n \geq 6$ LPS-treated group). (d) Bone marrow chimeras (donor $\rightarrow$ recipient) were challenged i.n. with LPS ( $0.5 \mathrm{mg} \mathrm{kg}{ }^{-1}$ of body weight). Six hours post challenge, the number of $\mathrm{Gr} 1^{+}$cells in the BAL was determined by flow cytometry $\left(n=8\right.$ per group). ${ }^{\star} P<0.05,{ }^{\star \star} P<0.01$,

${ }^{\star \star \star} P<0.005$, NS, not significant by Mann-Whitney U-test (a-c) or Kruskal-Wallis test with Dunn's multiple comparisons test (d).

Evans blue leakage into the lungs compared with WT $(\mathrm{C} 57 \mathrm{BL} / 6 \mathrm{~N})$ mice, suggesting diminished vascular leakage in response to inflammatory stimuli (Fig. 2c).

We next sought to determine whether the defect in neutrophil migration in Nlrp12-/ mice was due to the loss of NLRP12 in the haematopoietic compartment. We generated bone marrow chimeric mice in which NLRP12 deficiency was restricted to either the haematopoietic or non-haematopoietic compartment. WT $(\mathrm{C} 57 \mathrm{BL} / 6 \mathrm{~N})$ mice that received Nlrp12-/- bone marrow had defective neutrophil recruitment to the lungs in response to an i.n. LPS challenge and recapitulated the phenotype observed in NLRP12-deficient mice (Fig. 2d). Conversely, Nlrp12-/- mice that received WT $(\mathrm{C} 57 \mathrm{BL} / 6 \mathrm{~N})$ bone marrow had intact neutrophil recruitment to the lungs in response to an i.n. LPS challenge in comparison to WT $(\mathrm{C} 57 \mathrm{BL} / 6 \mathrm{~N})$ mice that received WT (C57BL/6N) bone marrow (Fig. 2d). These results suggest that defective neutrophil recruitment in Nlrp12-/- mice was due to a deficiency of NLRP12 within the haematopoietic compartment.

Neutrophil extrinsic NLRP12 regulates neutrophil migration. To determine if neutrophil intrinsic Nlrp12 expression was required for appropriate neutrophil recruitment, we utilized an adoptive transfer model. In all, $5 \times 10^{6} \mathrm{CD} 45.1^{+} \mathrm{WT}$ (confirmed to be $N \operatorname{lrp} 12^{B 6 N / B 6 N}$ ) bone marrow cells, as a source of neutrophils, were transferred intravenously into CD45.2 ${ }^{+}$WT $(\mathrm{C} 57 \mathrm{BL} / 6 \mathrm{~N})$ or $\mathrm{CD} 45.2^{+} \mathrm{Nlrp12} 2^{-/}$mice that were then challenged i.n. with LPS. Six hours later the number of CD45.1 ${ }^{+}$ neutrophils in the BAL fluid was quantified by flow cytometry (Fig. 3a). As expected, the total number of neutrophils recovered from the BAL of Nlrp12 $12^{-/}$mice was diminished compared with that from WT $(\mathrm{C} 57 \mathrm{BL} / 6 \mathrm{~N})$ recipient mice (Fig. 3b). In addition, the number of CD45.1 $1^{+} \mathrm{WT}\left(N \operatorname{lrp} 12^{B 6 N / B 6 N}\right)$ donor neutrophils in the BAL of $N l r p 12^{-/-}$recipient mice was also significantly diminished (Fig. 3c), indicating that normal $\mathrm{CD} 45.1^{+} \mathrm{WT}\left(\mathrm{Nlrp} 12^{B 6 N / B 6 N}\right)$ donor neutrophils exhibited defective recruitment in an NLRP12-deficient environment. To confirm that the donor CD45.1 ${ }^{+}$neutrophils had not been specifically eliminated in the Nlrp $12^{-/-}$recipient mouse, we compared the frequency of $\mathrm{CD} 45.1^{+}$donor neutrophils in the peripheral blood of WT $(\mathrm{C} 57 \mathrm{BL} / 6 \mathrm{~N})$ and $N l r p 12^{-/}$recipient mice. A similar frequency of $\mathrm{CD} 45.1^{+}$neutrophils were measured in the blood of both WT (C57BL/6N) and Nlrp12-Irecipient mice (Fig. $3 \mathrm{~d}$ ).

To confirm these findings, we performed the complementary experiment wherein $5 \times 10^{6} \mathrm{CD} 45.2^{+}$bone marrow cells from WT $(\mathrm{C} 57 \mathrm{BL} / 6 \mathrm{~N})$ or Nlrp12-/- mice were transferred intravenously into $\mathrm{CD} 45.1^{+} \mathrm{WT}\left(\mathrm{Nlrp} 12^{B 6 N / B 6 N}\right)$ mice. Mice were challenged i.n. with LPS and $6 \mathrm{~h}$ later the number of donor CD $45.2^{+}$neutrophils in the BAL fluid was quantified by flow cytometry (Fig. 3e). The total number of neutrophils recovered 
a
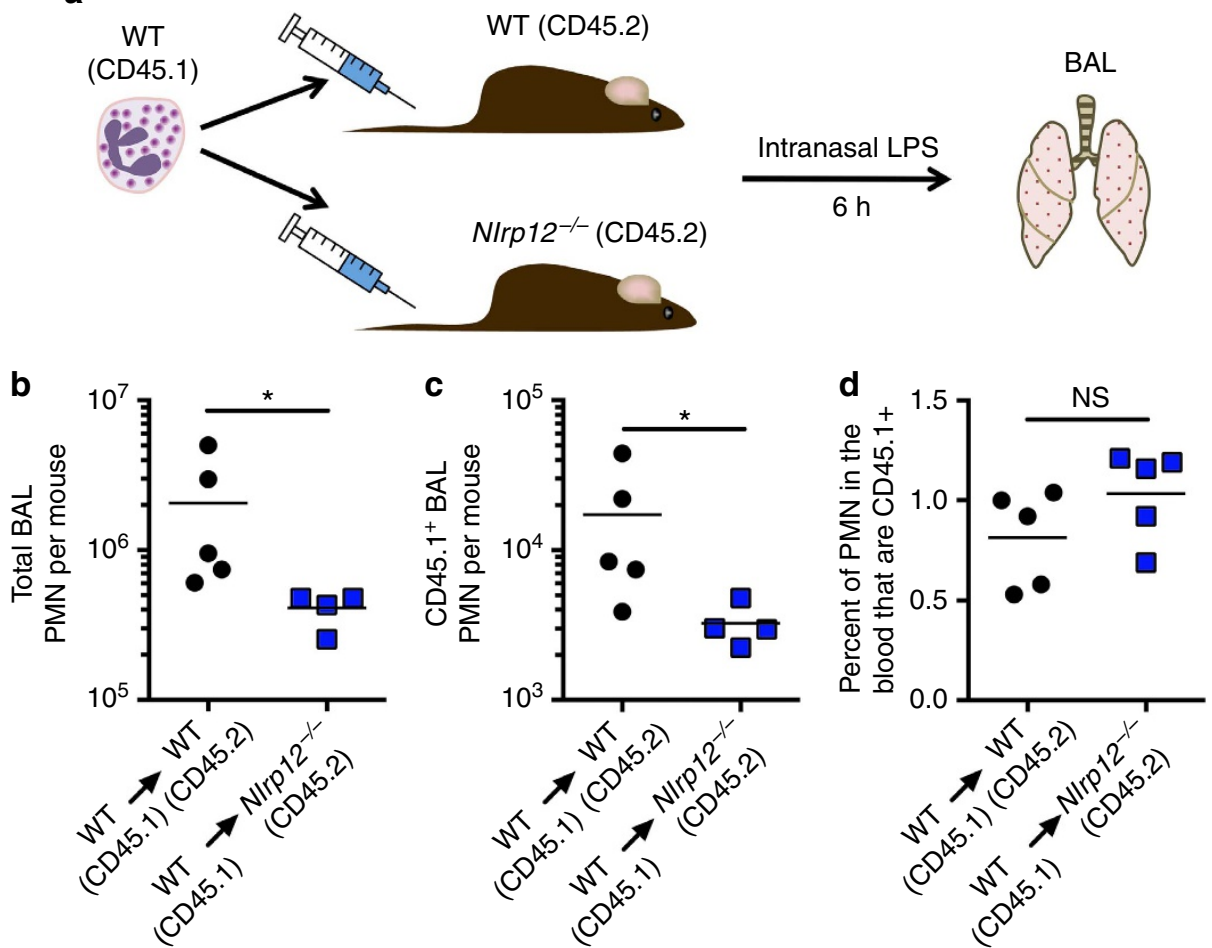

e
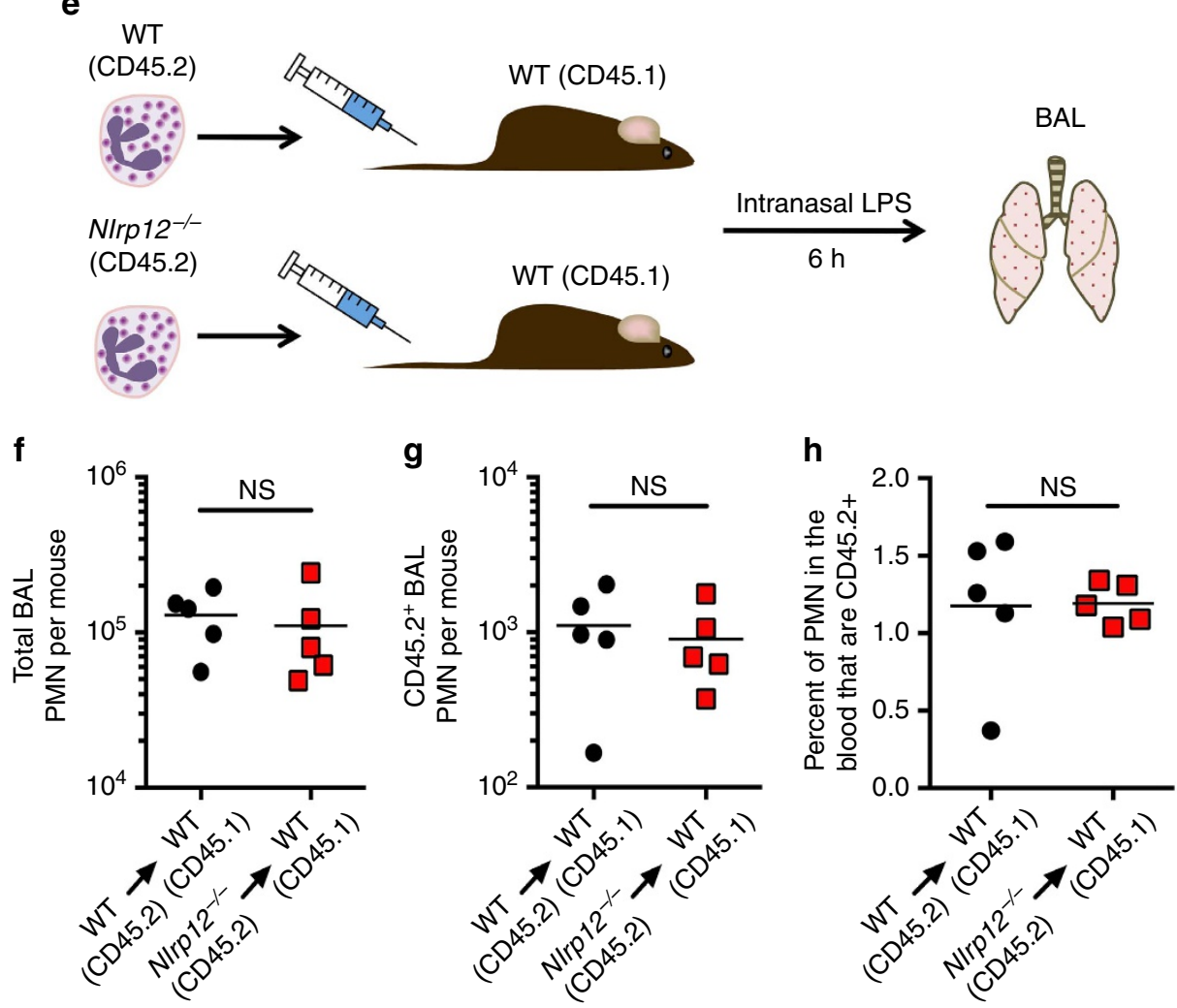

Figure 3 | The requirement for NLRP12 in neutrophil recruitment is neutrophil extrinsic. (a-d) A schematic illustrating the experimental approach is shown (a). WT CD45.1 ${ }^{+}$bone marrow was adoptively transferred into CD45.2+ WT or CD45.2+ N/rp12-/- mice. Mice were challenged i.n. with LPS ( $0.5 \mathrm{mg} \mathrm{kg}{ }^{-1}$ of body weight); $6 \mathrm{~h}$ post challenge the total number of $\mathrm{Gr} 1^{+}$cells $(\mathbf{b})$ and the number of $\mathrm{CD} 45.1^{+} \mathrm{Gr} 1^{+}$cells $(\mathbf{c})$ in the BAL was determined by flow cytometry. The percentage of $\mathrm{CD} 45.1^{+} \mathrm{Gr} 1^{+}$cells in the blood was also measured by flow cytometry (d). (e-h) A schematic illustrating the experimental approach is shown e. CD45.2 ${ }^{+}$WT or CD45.2 $2^{+} \mathrm{N} / r p 12^{-/-}$bone marrow was adoptively transferred into CD45.1 ${ }^{+}$WT mice. Mice were challenged i.n. with LPS $\left(0.5 \mathrm{mg} \mathrm{kg}{ }^{-1}\right.$ of body weight); $6 \mathrm{~h}$ post challenge the total number of $\mathrm{Gr} 1^{+}$cells $(\mathbf{f})$ and the number of CD45.2 $2^{+} \mathrm{Gr} 1^{+}$cells (g) in the BAL was determined by flow cytometry. The percentage of CD45.2 ${ }^{+} \mathrm{Gr}^{+}{ }^{+}$cells in the blood was also measured by flow cytometry (h). Data are representative of two independent experiments. ${ }^{\star} P<0.05$, NS not significant by Mann-Whitney $U$-test. 
from the $\mathrm{BAL}$ of $\mathrm{CD} 45.1^{+} \mathrm{WT}\left(\mathrm{Nlrp12} 2^{\mathrm{B} 6 \mathrm{~N} / \mathrm{B} 6 \mathrm{~N}}\right)$ mice that had received bone marrow cells from either WT $(\mathrm{C} 57 \mathrm{BL} / 6 \mathrm{~N})$ or Nlrp12-l- mice was similar (Fig. 3f). Moreover, we saw no difference in the number of donor CD45.2 ${ }^{+}$WT $(\mathrm{C} 57 \mathrm{BL} / 6 \mathrm{~N})$ or Nlrp12-/- neutrophils in the BAL of CD45.1 ${ }^{+}$WT $\left(N \operatorname{lrp} 12^{B 6 N / B 6 N}\right)$ recipient mice or in the frequency of donor CD45.2 ${ }^{+}$WT $(\mathrm{C} 57 \mathrm{BL} / 6 \mathrm{~N})$ and Nlrp12 $2^{-/-}$neutrophils in the peripheral blood of CD45.1 ${ }^{+} \mathrm{WT}\left(\mathrm{Nlrp} 12^{\mathrm{B} 6 \mathrm{~N} / \mathrm{B} 6 \mathrm{~N}}\right)$ recipient mice (Fig. 3g,h). These data suggest that Nlrp12 expression is required in a haematopoietic cell extrinsic to the neutrophil to appropriately direct neutrophil migration to inflammatory stimuli.

Macrophage NLRP12 is critical for neutrophil recruitment. Based on our finding that NLRP12 extrinsic to neutrophils modulated neutrophilic recruitment to inflammatory sites, we hypothesized that macrophage expression of NLRP12 might instruct neutrophil migration indirectly. To examine this hypothesis, we transferred WT (C57BL/6N) or NLRP12-deficient bone marrow-derived macrophages (BMDM) i.n. into WT (C57BL/6N) or Nlrp12-/- mice. Sixteen hours later mice were challenged i.n. with LPS and then the neutrophilic influx was determined in the BAL fluid (Fig. 4a,b). Transfer of WT (C57BL/6N), but not NLRP12-deficient, BMDM into Nlrp12-/mice rescued the neutrophil migration defect in vivo in response to LPS challenge (Fig. 4b). These data suggest that NLRP12 expression in macrophages is required to direct appropriate neutrophil migration in vivo.

Next, we applied the findings from the NLRP12-deficient mice to $\mathrm{C} 57 \mathrm{BL} / 6 \mathrm{~J}$ mice that carry a missense polymorphism in Nlrp12. To determine if the mechanism for the diminished neutrophil recruitment in $\mathrm{C} 57 \mathrm{BL} / 6 \mathrm{~J}$ mice is also through defective macrophage function we transferred WT $(\mathrm{C} 57 \mathrm{BL} / 6 \mathrm{~N})$ or Nlrp12 ${ }^{-1-}$ BMDM i.n. into C57BL/6N and C57BL/6J recipient mice. Sixteen hours later mice were challenged i.n. with LPS and neutrophil influx determined in the BAL fluid. Transfer of WT (C57BL/6N) BMDM into C57BL/6J recipient mice resulted in the rescue of neutrophil recruitment in response to i.n.
LPS as compared with WT (C57BL/6N) recipient mice (Fig. 4c). Importantly, transfer of NLRP12-deficient BMDM into C57BL/6J mice failed to rescue the neutrophil migration defect in vivo in response to LPS challenge (Fig. 4c), suggesting that defective NLRP12 function in C57BL/6J macrophages is responsible for the diminished neutrophil recruitment to inflammatory insults in vivo in these mice.

Nlrp12 ${ }^{-/-}$mice are more susceptible to bacterial infections. To determine if the defective neutrophil recruitment observed in Nlrp12-I- mice affected their ability to control bacterial infections in vivo, analogous to the C57BL/6J mice with the missense polymorphism in Nlrp12, we utilized a pulmonary F. tularensis LVS infection model. ASC-, NLRP3-, NLRP6- and NLRP12-deficient mice were challenged i.n. with a sub-lethal inoculum of $F$. tularensis LVS and survival monitored. As previously reported, mice deficient in ASC (a critical component of several inflammasomes including the AIM2 inflammasome) were highly susceptible to infection with $F$. tularensis LVS with all ASC-deficient mice succumbing to infection by day 13 post infection (Fig. 5a; (refs 17,18). However, NLRP3- or NLRP6-deficiency did not compromise the ability of mice to survive an i.n. infection with $F$. tularensis LVS (Fig. 5a). In contrast, but consistent with the results for C57BL/6J mice (Fig. 1c), NLRP12-deficient mice were highly susceptible to i.n. infection with $F$. tularensis LVS with $>75 \%$ of NLRP12-deficient mice succumbing to infection by day 12 post infection (Fig. $5 \mathrm{a}$ ). The increased susceptibility of Nlrp12 $12^{-/}$mice to infection with F. tularensis LVS compared with WT $(\mathrm{C} 57 \mathrm{BL} / 6 \mathrm{~N})$ mice was also reflected by increased bacterial burdens in infected organs 6 and 9 days post infection (Fig. 5b and Supplementary Fig. 3). NLRP12-deficient mice were also more susceptible to an intraperitoneal route of infection with $F$. tularensis LVS compared with WT mice, suggesting that the role of NLRP12 in host defense is not restricted to the lung (Fig. 5c).

In order to determine if NLRP12 had a more global role in host defense against pathogens, we infected NLRP12-deficient mice
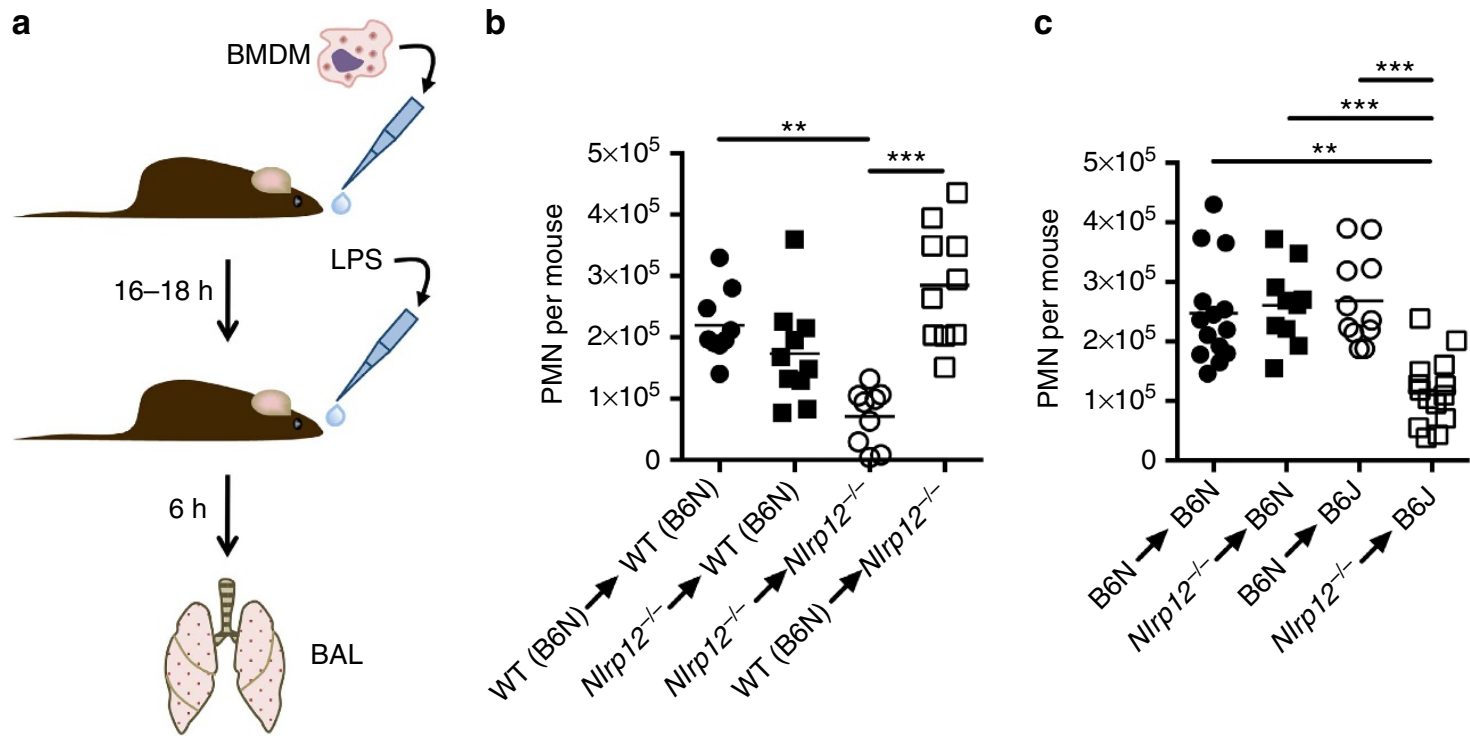

Figure 4 | WT (B6N) macrophages rescue the neutrophil defect in C57BL/6J and N/rp12-/- mice. (a) A schematic illustrating the experimental approach is shown. (b) In all, $4 \times 10^{6}$ BMDM from WT (B6N) or Nlrp12-/- mice were transferred i.n. into either WT (B6N) or N/rp12-/- mice (donor $\rightarrow$ recipient). (c) In all, $4 \times 10^{6}$ BMDM from WT (B6N) or Nlrp12-/- mice were transferred i.n. into either WT (B6N) or WT (B6J) mice (donor $\rightarrow$ recipient). $(\mathbf{b}, \mathbf{c})$ Mice were rested overnight and then challenged i.n. with LPS $\left(0.5 \mathrm{mg} \mathrm{kg}^{-1}\right.$ of body weight). Six hours post challenge the number of $\mathrm{Gr} 1^{+}$cells in the BAL was determined by flow cytometry ( $n \geq 8$ per group). ${ }^{\star \star} P<0.01,{ }^{\star \star \star} P<0.005$ by Kruskal-Wallis test with Dunn's multiple comparisons test. 
a
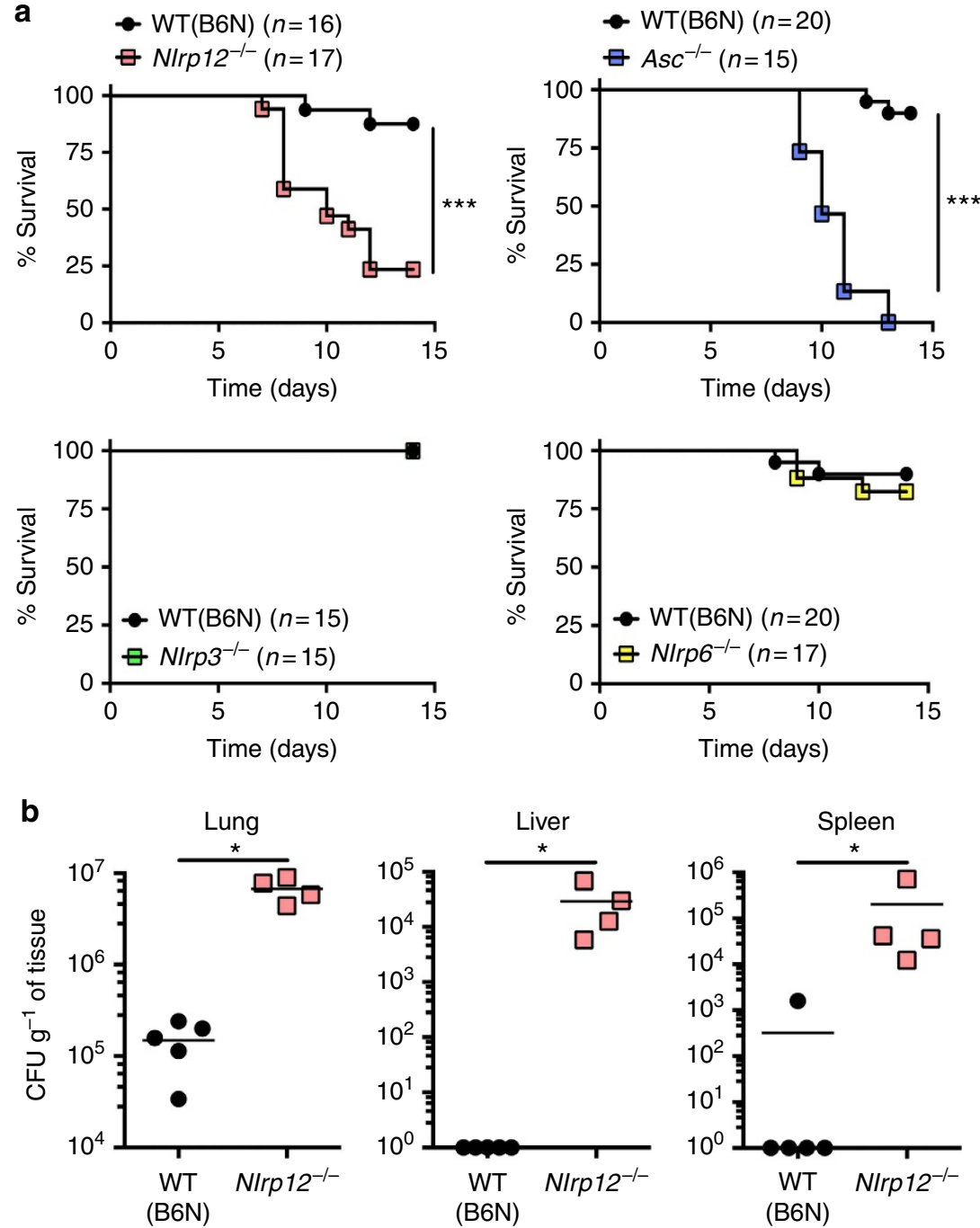

C
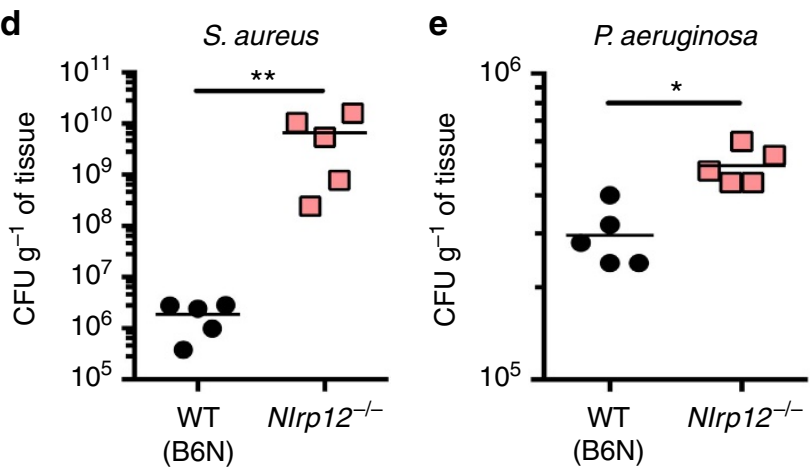

Figure 5 | NLRP12 is required for effective control of bacterial pathogens in vivo. (a) WT (B6N), NLRP12-, NLRP3-, NLRP6- and ASC-deficient mice were infected i.n. with $5 \times 10^{3}$ colony-forming unit (CFU) of $F$. tularensis LVS and survival monitored. (b) Nine days post infection with $5 \times 10^{3}$ CFU of $F$. tularensis LVS organs were harvested, homogenized and dilutions plated for enumeration of CFU. (c) WT (B6N) and Nlrp12-/- mice were infected intraperitoneally with $5 \times 10^{3} \mathrm{CFU}$ of $F$. tularensis LVS and survival monitored. (d,e) WT (B6N) and NIrp12-/- mice were infected i.n. with either $5 \times 10^{7}$ CFU of S. aureus Newman strain (d) or $5 \times 10^{4}$ CFU of $P$. aeruginosa PAK strain (E). Sixteen hours (for $S$. aureus infection) or $4 \mathrm{~h}$ (for $P$. aeruginosa infection) post infection lungs were harvested, homogenized and dilutions plated for enumeration of CFU. Pooled data from 2-3 independent experiments is shown; ${ }^{\star} P<0.05$, ${ }^{\star \star \star} P<0.005$ by log-rank test $(\mathbf{a}, \mathbf{c})$. Data are representative of $2-3$ independent experiments; ${ }^{\star} P<0.05$, ${ }^{\star \star} P<0.01$ by Mann-Whitney $U$-test $(\mathbf{b}, \mathbf{d}, \mathbf{e})$.

i.n. with Staphylococcus aureus and Pseudomonas aeruginosa. Similar to the findings with $F$. tularensis LVS, Nlrp12-/- mice had significantly higher bacterial burdens of $S$. aureus and $P$. aeruginosa in their lungs compared with WT $(\mathrm{C} 57 \mathrm{BL} / 6 \mathrm{~N})$ mice (Fig. 5d,e). Taken together, these data suggest that NLRP12 has an important role in controlling not only intracellular
Gram-negative bacteria but also extracellular Gram-negative and Gram-positive pathogens.

Reduced neutrophil recruitment to F. tularensis in Nlrp12-/mice. Neutrophils are rapidly recruited to inflammatory sites 
through a number of well-defined steps ${ }^{19}$. Neutrophils are initially mobilized from the bone marrow into the peripheral circulation. After receiving the appropriate signals neutrophils interact with the endothelium and undergo tethering, rolling, adhesion, crawling and transendothelial migration. In some instances, neutrophils can additionally exit the tissue parenchyma via transepithelial migration. To define at what point neutrophil migration was disrupted in Nlrp12-I- mice, we evaluated the in vivo mobilization of inflammatory cells in response to infection with $F$. tularensis LVS. Circulating neutrophils were markedly increased at day 3 post infection in both WT $(\mathrm{C} 57 \mathrm{BL} / 6 \mathrm{~N})$ and Nlrp12 $12^{-1-}$ mice (Fig. 6a). This suggested two things: first that the absence of NLRP12 did not impact the development of neutrophils or second impact their release from the bone marrow into peripheral circulation. In contrast, significantly fewer neutrophils were recovered from the BAL fluid following infection with $F$. tularensis LVS in Nlrp12-/- mice compared with WT (C57BL/6N) (Fig. 6b and Supplementary Fig. 4).

Movement from the circulation into the alveolar space requires the neutrophils to cross first an endothelial and then an epithelial barrier. To differentiate if neutrophil migration was impaired at the level of transendothelial or transepithelial migration, we assessed the number of neutrophils in the lung parenchyma by flow cytometry. Analogous to the loss of neutrophils in the BAL fluid, we also observed a similar defect in the number of neutrophils recruited across the endothelial barrier into the lung parenchyma of $\mathrm{Nlrp} 12^{-/-}$mice 6 days post infection with F. tularensis LVS (Fig. 6c). The impact of NLRP12 deficiency was limited to the neutrophilic response, as the number of parenchymal macrophages and dendritic cells were not significantly different between WT (C57BL/6N) and $N \operatorname{lrp} 12^{-/-}$mice (Fig. 6c). Taken together, these data suggest

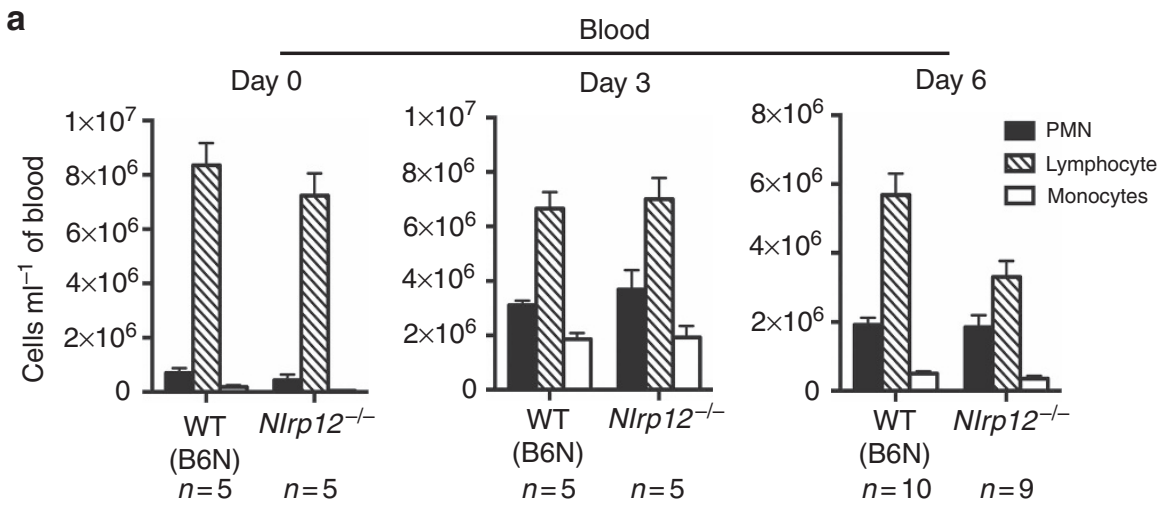

b

b Lung (BAL)

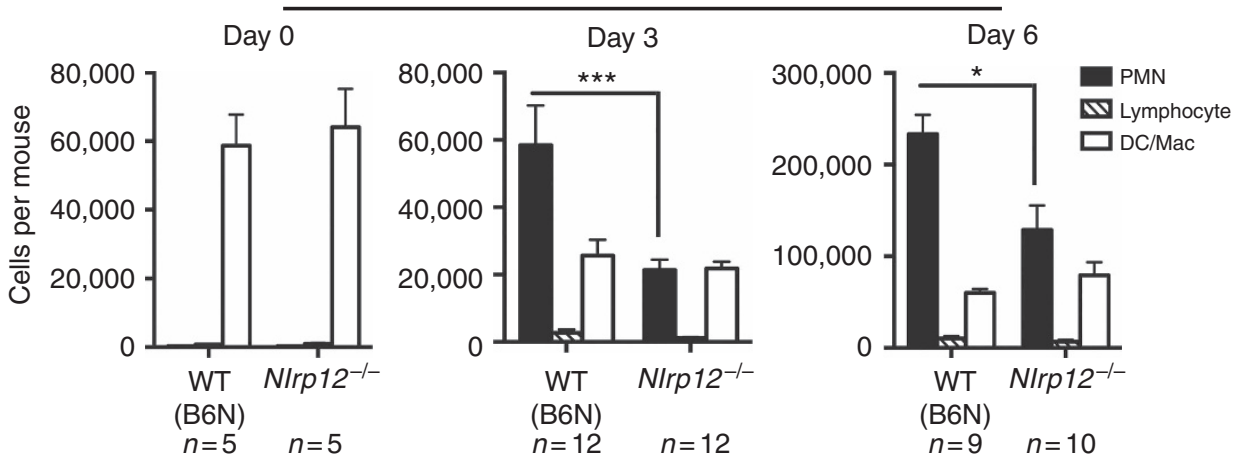

C

Lung (tissue)
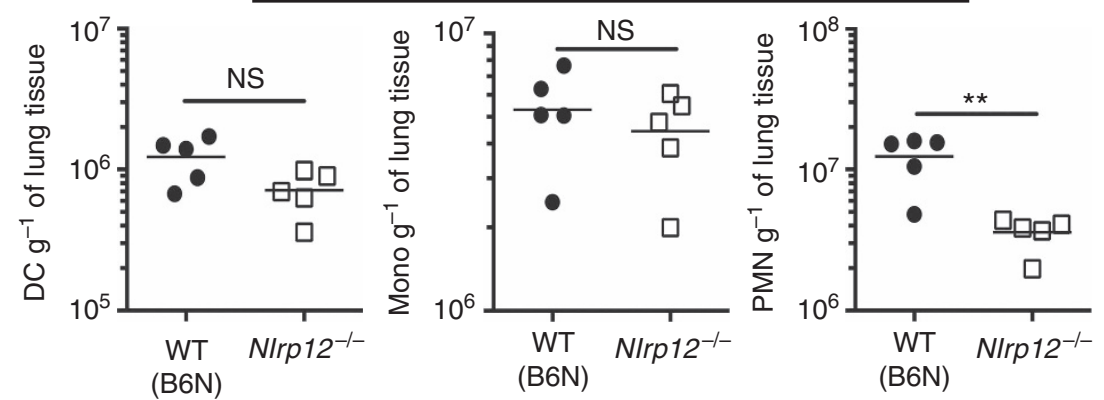

Figure 6 | NLRP12 is required for neutrophil recruitment following $\boldsymbol{F}$. tularensis infection. (a,b) WT (B6N) or N/rp12-/- mice were infected i.n. with $5 \times 10^{3}$ colony-forming unit (CFU) of $F$. tularensis LVS. At the indicated time post infection blood was collected and BAL performed. Cell count and differential on blood (a) and BAL (b) was performed. (c) Three days post i.n. infection with $5 \times 10^{3} \mathrm{CFU}$ of $F$. tularensis LVS lungs were harvested, prepared into single cell suspensions, stained and analysed by flow cytometry for dendritic cells (CD11b $\left.{ }^{+}, \mathrm{CD}_{11} \mathrm{c}^{\text {high }}, \mathrm{Gr}^{-}\right)$, macrophages/monocytes (CD11b $\left.{ }^{+}, \mathrm{CD}_{11} \mathrm{c}^{\text {low }}, \mathrm{Gr}^{-}{ }^{-}\right)$and

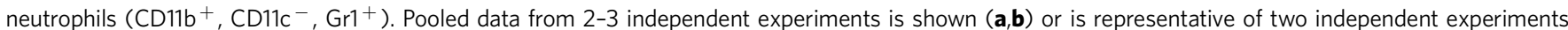
(c). Data are expressed as the mean \pm s.e.m. (a,b). ${ }^{\star} P<0.05,{ }^{\star \star} P<0.01,{ }^{\star \star \star} P<0.005, \mathrm{NS}$, not significant by Mann-Whitney $U$-test. 
that the defect in neutrophil migration observed in Nlrp12 $12^{-/-}$ mice infected with $F$. tularensis LVS lies at the level of transendothelial migration out of the vasculature and into the lung parenchyma.

NLRP12-deficient macrophages have defective CXCL1 production. To further characterize the role of NLRP12 in the response to $F$. tularensis LVS infection, we next asked what the mechanism is by which NLRP12 acts in macrophages to direct neutrophil migration. We examined the induction of cytokines and chemokines in BAL fluid from WT (C57BL/6N) and NLRP12deficient mice 3 days post infection. Whereas IL-6, tumour-necrosis factor (TNF $\alpha$ ), IL-17A, macrophage inflammatory protein (MIP)$1 \alpha$, IL-18 and IL-1 $\beta$ levels were not different in Nlrp12 ${ }^{-/-}$mice compared with WT (C57BL/6N) mice, the neutrophil chemotactic chemokine CXCL1 levels were significantly diminished in the BAL fluid from Nlrp12 $2^{-/-}$mice compared with WT (B6N) (Fig. 7a and Supplementary Fig. 5a).

Consistent with our in vivo findings of diminished CXCL1 in BAL from Nlpr12 $12^{-/}$mice following infection with F. tularensis LVS, we observed that $N$ lpr $12^{-/-}$BMDM secreted significantly less CXCL1 in response to $F$. tularensis LVS, S. aureus and P. aeruginosa (Fig. 7b). We observed a similar defect in CXCL1 production in the absence of NLRP12 in bone marrow-derived dendritic cells (BMDC) challenged with $F$. tularensis LVS, $S$. aureus or $P$. aeruginosa (Supplementary Fig. 5b). However, the production of IL- 6 , TNF $\alpha$ and IL-10 in response to infection with $F$. tularensis LVS, S. aureus or $P$. aeruginosa was
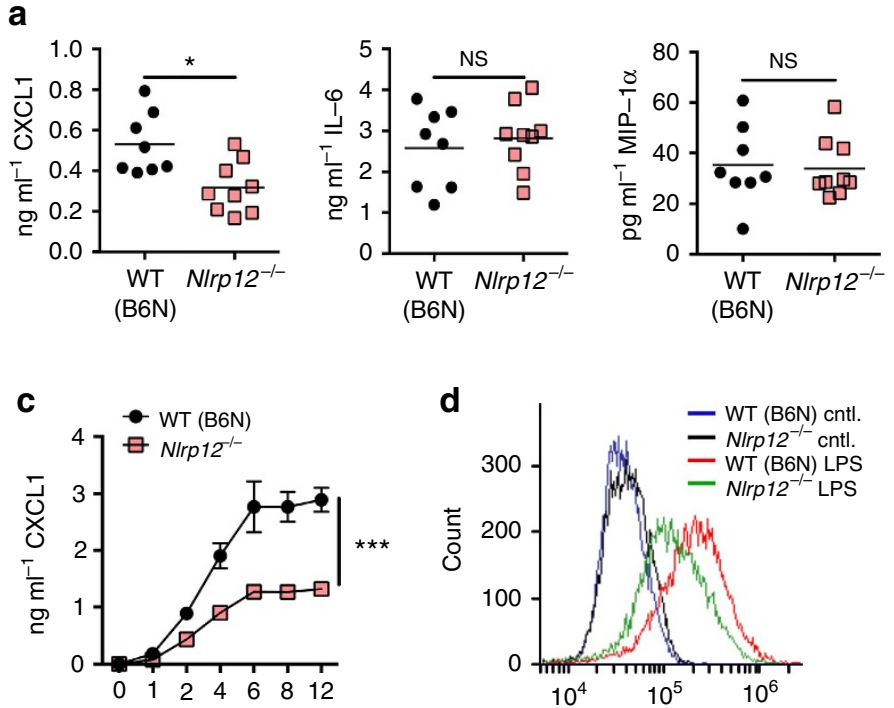

Hours post addition of LPS

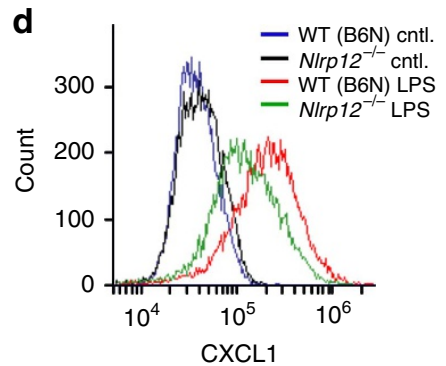

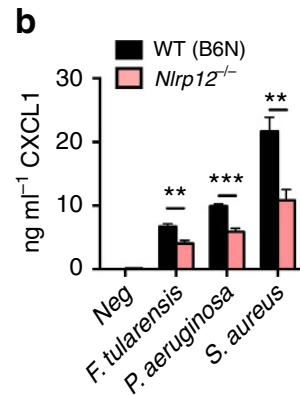
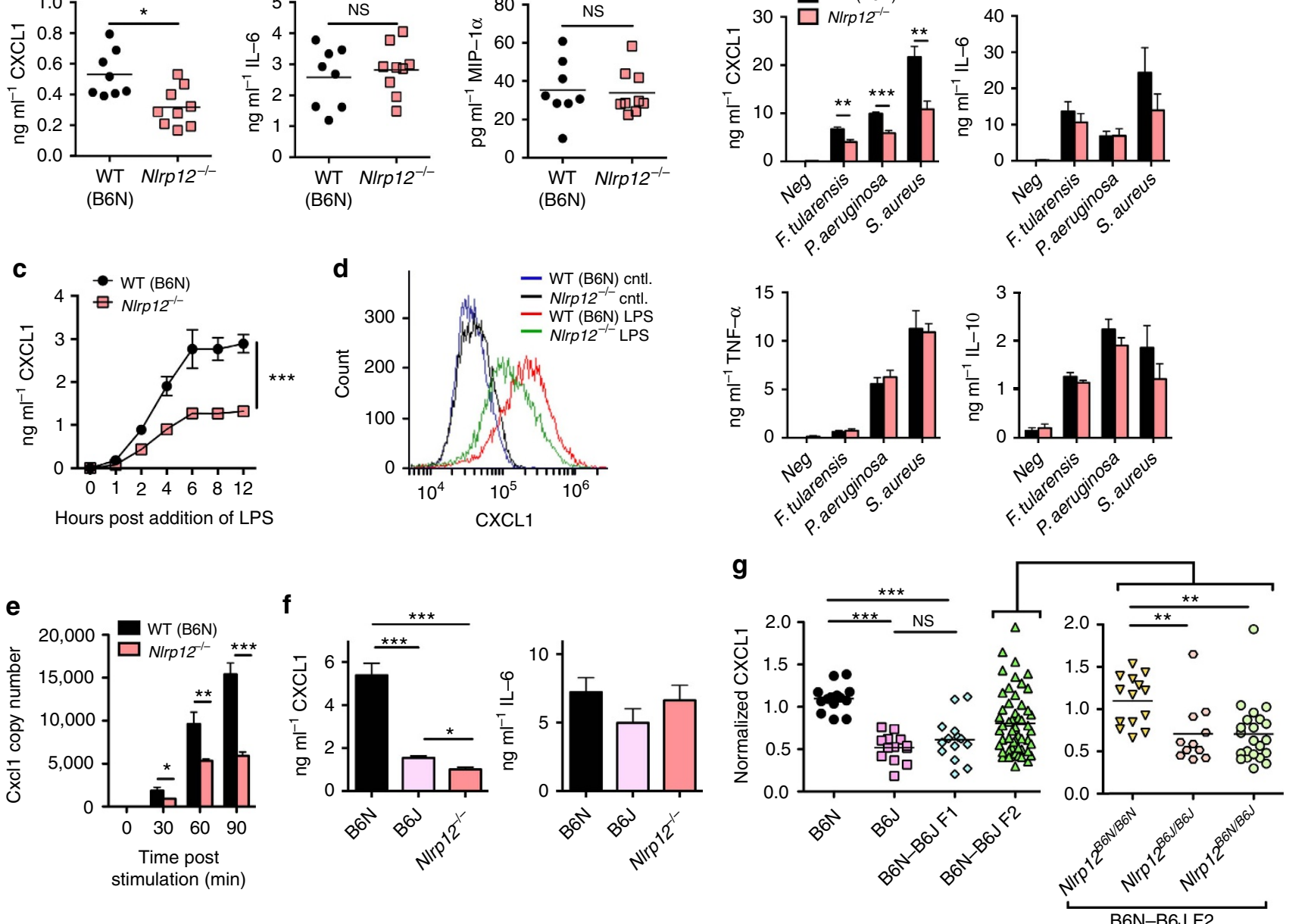

Figure 7 | BMDM from C57BL/6J and NIrp12 ${ }^{-/-}$mice have defective CXCL1 production. (a) WT (B6N) or N/rp12 ${ }^{-/-}$mice were infected i.n. with $5 \times 10^{3}$ colony-forming unit of $F$. tularensis LVS. Three days post infection BAL were performed and cytokine levels were determined by ELISA ( $n=8$, WT; $n=9$, NIrp12-/-). (b) BMDM from WT (B6N) and NIrp12-/- mice were challenged with either F. tularensis LVS, S. aureus or P. aeruginosa. Eight hours later supernatants were collected and secretion of the indicated cytokine or chemokine quantified by ELISA. (c) BMDM from WT (B6N) and N/rp12-/mice were stimulated with LPS $\left(50 \mathrm{ng} \mathrm{ml}^{-1}\right)$ for the indicated amount of time; supernatants were collected and assayed for CXCL1 production by ELISA. (d) BMDM from WT (B6N) and N/rp12-/- mice were left unstimulated or stimulated for $4 \mathrm{~h}$ with LPS in the presence of brefeldin A and monensin; intracellular CXCL1 was then assessed by flow cytometry. (e) BMDM from WT (B6N) and N/rp12-/- mice were stimulated with LPS for the indicated amount of time; Cxcl1 expression was quantified by real-time PCR. (f) BMDM from WT (B6N), WT (B6J) and Nlrp12-/- mice were stimulated with LPS for $8 \mathrm{~h}$; supernatants were collected and assayed for the indicated cytokines by ELISA. (g) BMDM from B6N, B6J, B6N-B6J F1 and B6N-B6J F2 mice were challenged with LPS for $8 \mathrm{~h}$; supernatants were collected and CXCL1 secretion assessed by ELISA. The N/rp12 allele was sequenced for all B6N-B6J F2 mice and cohorts stratified based on their N/rp12 genotype (N/rp12 ${ }^{B 6 N / B 6 N}$, Nlrp12 ${ }^{B 6 N / B 6 J}$ or N/rp12 $\left.{ }^{B 6 J / B 6 J}\right)(n=14, B 6 N ; n=13, B 6 J ; n=14, F 1 ; n=52, F 2)$. Pooled data from three $(\mathbf{b}, \mathbf{c})$ independent experiments are depicted or are representative of two $(\mathbf{d})$ or three $(\mathbf{e}, \mathbf{f})$ independent experiments. $(\mathbf{b}, \mathbf{c}, \mathbf{e}, \mathbf{f})$ Data are expressed as the mean \pm s.e.m. ${ }^{\star} P<0.05,{ }^{\star \star} P<0.01,{ }^{\star \star \star} P<0.005, N S$, not significant by Mann-Whitney $U$-test $(\mathbf{a}, \mathbf{g})$, Student's $t$-test $(\mathbf{b}, \mathbf{e}, \mathbf{f})$ or two-way analysis of variance analysis (c). 
similar between WT $(\mathrm{C} 57 \mathrm{BL} / 6 \mathrm{~N})$ and $\mathrm{Nlrp12}{ }^{-/-} \mathrm{BMDM}$ (Fig. 7b).

To determine if the defect in CXCL1 production was restricted to infection with viable bacteria, we challenged BMDM from WT $(\mathrm{C} 57 \mathrm{BL} / 6 \mathrm{~N})$ and Nlrp12 ${ }^{-/-}$mice with a variety of toll-like receptor (TLR) agonists. Whereas NLRP12-deficient BMDM produced amounts of IL- 6 and TNF $\alpha$ similar to those of WT (C57BL/6N) BMDM, NLRP12-deficient cells released less CXCL1 in response to all TLR agonists tested (Fig. 7c and Supplementary Fig. 5c). In support of the enzyme-linked immunosorbent assay (ELISA) data, intracellular cytokine staining also demonstrated less CXCL1 production in NLRP12-deficient BMDM compared with that in WT $(\mathrm{C} 57 \mathrm{BL} / 6 \mathrm{~N})$ challenged with LPS (Fig. 7d). Examination of Cxcl1 expression by quantitative reverse transcription-PCR also found diminished Cxcl1 expression in NLRP12-deficient BMDM compared with WT (C57BL/6N) BMDM stimulated with LPS (Fig. 7e). Evaluation of mitogenactivated protein kinases (MAPK), canonical NF- $\mathrm{KB}$ and non-canonical NF- $\kappa B$ pathways in response to LPS stimulation did not reveal any substantial differences between BMDM from WT (C57BL/6N) and Nlrp12 $2^{-/}$mice (Supplementary Fig. $5 \mathrm{~d}-\mathrm{g}$ ). Taken together, these data suggest that NLRP12 was specifically required for CXCL1 production and that this regulation occurred at the level of Cxcl1 transcript abundance.

Vladimer et al. ${ }^{10}$ have shown that $Y$. pestis can activate macrophage caspase-1 in an NLRP12-dependent manner and result in the generation of IL-1 $\beta$ and IL-18. To determine if NLRP12 was required to trigger inflammasome activation in response to $F$. tularensis LVS, S. aureus or $P$. aeruginosa, we measured IL-1 $\beta$ secretion and caspase-1 activation from BMDM from WT $(\mathrm{C} 57 \mathrm{BL} / 6 \mathrm{~N})$ and $\mathrm{Nlrp} 12^{-/-}$mice challenged with $F$. tularensis LVS, S. aureus or P. aeruginosa. The production of IL- $1 \beta$ and caspase-1 activation between WT $(\mathrm{C} 57 \mathrm{BL} / 6 \mathrm{~N})$ and NLRP12-deficient BMDM following challenge with $F$. tularensis LVS, S. aureus or P. aeruginosa were similar (Supplementary Fig. $6 a, b)$. Similarly, no defect in the production of IL-1 $\beta$ in the absence of NLRP12 was observed in BMDC challenged with F. tularensis LVS, S. aureus or P. aeruginosa (Supplementary Fig. 6c). These data demonstrate that NLRP12 did not participate in the assembly or activation of an inflammasome in response to F. tularensis LVS, S. aureus or $P$. aeruginosa, and that the increased susceptibility of $\mathrm{Nlrp12} \mathrm{I}^{-{ }_{-}}$mice to infection with these organisms was independent of caspase-1 activation.

Diminished CXCL1 production in C57BL/6J macrophages. We next examined if BMDM from C57BL/6J also exhibited defects in CXCL1 production in response to LPS. Similar to Nlrp12 ${ }^{-/-}$ BMDM, CXCL1 production from C57BL/6J BMDM stimulated with LPS was significantly diminished compared with C57BL/6N BMDM. Furthermore, CXCL1 production from C57BL/6J BMDM was diminished to a similar level as BMDM from Nlrp12 $2^{-/}$mice (Fig. 7f). Again consistent with the findings for Nlrp12-/- BMDM, in response to LPS stimulation C57BL/6J and $\mathrm{C} 57 \mathrm{BL} / 6 \mathrm{~N}$ BMDM produced equivalent levels of IL-6, TNF $\alpha$, IL- $1 \alpha$ and IL-10 (Fig. 7f and Supplementary Fig. 7a). Examination of Cxcl1 expression by quantitative reverse transcription-PCR also found diminished Cxcl1 expression in C57BL/6J BMDM compared with C57BL/6N BMDM stimulated with LPS (Supplementary Fig. 7b). Furthermore, LPS stimulation of TLR4-expressing HEK293 cells (HEK-TLR4) transfected with $\mathrm{Nlrp} 12^{\mathrm{BCN}}$ resulted in significantly greater CXCL1 production compared with stimulation of those transfected with Nlrp12 $2^{B 6 J}$ (Supplementary Fig. 7c,d).

To determine if the missense mutation in C57BL/6J Nlrp12 contributed to the diminished CXCL1 production observed in
C57BL/6J BMDM we analysed CXCL1 production from BMDM in response to LPS from $52 \mathrm{~F}_{2}$ offspring that were then segregated by Nlrp12 genotype (Fig. $7 \mathrm{~g}$ ). The presence of the C57BL/6J missense polymorphism in Nlrp12 correlated with diminished CXCL1 production by the $\mathrm{F}_{2}$ BMDM (Fig. $7 \mathrm{~g}$ ). BMDM from $\mathrm{F}_{1}$ mice and $\mathrm{F}_{2}$ mice that were heterozygous at the Nlrp12 locus had CXCL1 production similar to C57BL/6J mice in response to LPS challenge, further suggesting the dominance of the C57BL/6J trait (Fig. $7 \mathrm{~g}$ ).

\section{Discussion}

Our work demonstrates differences in inflammatory responses between C57BL/6N and C57BL/6J mouse substrains with C57BL/6J mice having a failure of neutrophil recruitment to a wide variety of inflammatory stimuli compared with the related C57BL/6N substrain. C57BL/6J mice carry a missense polymorphism in chromosome 7 at position $3,222,537$ (G to A) leading to the change of an arginine for lysine in the C-terminal leucine-rich repeat domain of NLRP12 (ref. 1). Our findings show that this Nlrp12 SNP is associated with the difference in neutrophil recruitment found between $\mathrm{C} 57 \mathrm{BL} / 6 \mathrm{~N}$ and $\mathrm{C} 57 \mathrm{BL} / 6 \mathrm{~J}$ substrains and that NLRP12 is required in macrophages to direct neutrophil recruitment. This failure by macrophages, both the NLRP12-deficient and the NLRP12 missense mutant, to recruit neutrophils is associated with defective production of the neutrophil chemoattractant chemokine CXCL1. These data suggest a single seemingly conservative amino acid change within NLRP12 can render it dysfunctional and profoundly impact a wide range of immune responses both in vitro and in vivo. Our data suggest that C57BL/6J mice carrying the Nlrp12 missense polymorphism may be inappropriate controls to study NLRP12 function. Furthermore, the use of C57BL/6J mice, as well as knockout mice backcrossed onto the C57BL/6J substrain, in studies that depend upon intact neutrophilic responses or that assess control of infectious pathogens may significantly confound their interpretation due to the presence of a dysfunctional NLRP12.

The literature surrounding NLRP12 is complex as several lines of investigation suggest contradictory roles for NLRP12 in response to infection with bacterial pathogens. NLRP12 has been shown to be important for host defense against $Y$. pestis through the production of caspase-1-dependent IL-18 (ref. 10). In contrast, NLRP12 has a detrimental role in infection with $S$. typhimurium through negative regulation of $\mathrm{NF}-\mathrm{\kappa B}$ and MAPK $^{11}$. In the current study, we demonstrate a novel role for NLRP12 in host defense against infection with F. tularensis LVS, $S$. aureus and $P$. aeruginosa. In contrast to the findings with $Y$. pestis $^{10}$, we did not observe a defect in caspase- 1 mediated IL-1 $\beta$ production in Nlrp12 $2^{-/-}$mice in response to F. tularensis LVS, $S$. aureus and $P$. aeruginosa, suggesting that NLRP12 did not regulate inflammasome activation in response to these pathogens. However, Nlrp12-1- mice failed to control replication of $F$. tularensis LVS, S. aureus and P. aeruginosa in vivo, a failure that correlated with a defective recruitment of neutrophils to the site of infection. Mobilization of neutrophils from the bone marrow into the peripheral circulation was intact in the absence of NLRP12 in mice infected i.n. with F. tularensis LVS. However, these newly recruited neutrophils were defective in their ability to exit from the peripheral circulation and migrate into the lung parenchyma in Nlrp12 $2^{-/-}$mice, thereby suggesting a defect in transendothelial migration. Consistent with our findings a study by Cai et al. ${ }^{20}$ demonstrated that NLRP12-deficient mice were more susceptible to pulmonary infection with $K$. pneumoniae in vivo and that this was in part due to defective CXCL1 production. Interestingly, a study by Arthur et al. ${ }^{21}$ demonstrated 
an in vitro migration defect of NLPR12-deficient neutrophils to CXCL1. In contrast, Zamoshnikova et al. ${ }^{22}$ demonstrated an increase in neutrophil migration in vitro to the higher of two doses of CXCL1, but not in migration to Leishmania major. Although our in vivo assays did not reveal a neutrophil intrinsic role for NLRP12, the findings of Arthur et al. and Zamoshnikova et al. suggest there may be an additional neutrophil intrinsic role for NLRP12 (refs 21,22).

Perivascular macrophages in a variety of tissues are in close proximity to blood vessels and potently express CXCL1 and CXCL2 in response to challenge with infectious stimuli ${ }^{23}$. Chemokines secreted by perivascular macrophages are thought to create the precise cytokine milieu triggering both the adhesion and transmigration of neutrophils out of the blood stream ${ }^{23}$. Consistent with a role for a perivascular macrophage-like cell, local pulmonary reconstitution of Nlrp12-/- mice with WT $(\mathrm{C} 57 \mathrm{BL} / 6 \mathrm{~N})$ macrophages, but not Nlrp12 ${ }^{-/-}$ macrophages, corrected defective neutrophil recruitment to the lungs in response to LPS. These findings suggest that the NLRP12-dependent production of a chemotactic signal from local macrophages is a necessity for protective neutrophil recruitment in response to inflammatory stimuli in vivo. In further support of this role for macrophage-derived NLRP12, we observed a specific defect in the ability of NLRP12-deficient macrophages to secrete CXCL1 in response to a variety of pathogens and TLR agonists. These findings are consistent with a previous study in which diminished CXCL1 production was observed in NLRP12-deficient dendritic cells stimulated with the TLR2 agonist Pam3Cys4 compared with WT dendritic cells ${ }^{8}$.

In conclusion, our results demonstrate that NLRP12 is critical in the regulation of neutrophil recruitment to inflammatory triggers and that this is through control of the production of CXCL1 by local tissue macrophages. This neutrophil-extrinsic mechanism in turn is important for host defense against a variety of bacterial pathogens. Furthermore, a missense mutation in Nlrp12 in C57BL/6J mice renders them with defective NLRP12 function and hence diminished neutrophil recruitment to inflammatory stimuli in vivo. It is important to note that although the mutation in Nlrp12 in C57BL/6J mice results in defective neutrophil migration in vivo, it remains to be seen if other roles for NLRP12 are affected by this missense mutation. Further studies are also needed to define how the C57BL/6J missense mutation in NLPR12 affects its structure and binding to potential ligands and downstream signalling molecules. In addition, the relevance of these findings to human NLRP12 function will require further investigation.

\footnotetext{
Methods

Mice. The generation of NLRP12-, NLRP6-, NLRP3- and ASC-deficient mice has been described previously ${ }^{21,24,25}$. Mice were backcrossed onto the C57BL/6N genetic background for at least nine generations. Nlrp12 $2^{-/-}$mice were backcrossed onto a $\mathrm{BALB} / \mathrm{cJ}$ background for eight generations. C57BL/6N mice were purchased from National Cancer Institute and used as WT controls unless otherwise stated; $\mathrm{BALB} / \mathrm{cJ}$ and $\mathrm{C} 57 \mathrm{BL} / 6 \mathrm{NJ}$ mice were purchased from Jackson Laboratories. CD45.1 ${ }^{+}$C57BL/6 mice (B6-Ly5.2/Cr) were purchased from National Cancer Institute. Both male and female mice between 6 and 12 weeks of age were used, however individual experiments utilized age and sex matched animals. The Institutional Animal Care and Use Committee at the University of Iowa approved all protocols used in this study. Bone marrow chimeras were generated as described ${ }^{26}$. Reconstitution was $>92 \%$ in Nlrp12 $2^{-/-}$mice reconstituted with WT bone marrow and $89 \%$ in WT mice reconstituted with Nlrp12-/- bone marrow.
}

Bacterial strains and growth conditions. F. tularensis ssp. holarctica LVS was obtained from American Type Culture Collection (ATCC 29684). F. tularensis LVS was grown on Difco cysteine heart agar supplemented with $9 \%$ sheep red blood cells (SRBC) for $48 \mathrm{~h}$ at $37^{\circ} \mathrm{C}$. For in vivo studies, bacteria were grown overnight in modified Mueller-Hinton (MMH) broth (Becton Dickinson) supplemented with $1 \%(\mathrm{wt} / \mathrm{vol}$ ) glucose, $0.025 \%$ ferric pyrophosphate, and $2 \%$ IsoVitaleX and then subcultured for $4 \mathrm{~h}$ before use. F. tularensis LVS expressing green fluorescent protein (GFP) ${ }^{27}$ were grown on Difco cysteine heart agar supplemented with $9 \%$ SRBC and $25 \mu \mathrm{g} \mathrm{ml}^{-1}$ spectinomycin for $48 \mathrm{~h}$ at $37^{\circ} \mathrm{C}$; bacteria were then cultured in supplemented MMH broth with $25 \mu \mathrm{g} \mathrm{ml}{ }^{-1}$ spectinomycin at $37^{\circ} \mathrm{C}$ overnight, and subcultured for $4 \mathrm{~h}$ in supplemented MMH broth. P. aeruginosa PAK strain was obtained from Barbara Kazmierczak (Yale University). P. aeruginosa was cultured in lysogeny broth broth overnight at $37^{\circ} \mathrm{C}$ and then subcultured for $4 \mathrm{~h}$ before use. S. aureus Newman strain was obtained from William Nauseef (University of Iowa); S. aureus was cultured overnight in tryptic soy broth at $37^{\circ} \mathrm{C}$ and then subcultured for $4 \mathrm{~h}$ before use.

In vivo infection and LPS challenge. For in vivo infections mice were anaesthetized with ketamine/xylazine and infected i.n. with the indicated number of bacteria resuspended in $50 \mu \mathrm{l}$ of PBS. Alternatively, mice were injected intraperitoneally with $5 \times 10^{3}$ colony-forming unit of $F$. tularensis LVS resuspended in $200 \mu \mathrm{l}$ of PBS. Mice were monitored every $12-24 \mathrm{~h}$ for lethality; mice found to be in a moribund state for more than $4 \mathrm{~h}$ were considered terminal and euthanized. Bacterial burdens in the lung, spleen and liver of infected mice were determined at the indicated times post infection by dilution plating of tissue homogenates onto Difco cysteine heart agar supplemented with 9\% SRBC for F. tularensis LVS, lysogeny broth for P. aeruginosa or TSA for S. aureus. For in vivo LPS challenge mice were anaesthetized with ketamine/xylazine and challenged i.n. with the indicated amount of LPS (E. coli serotype 0111:B4; Invivogen) in $50 \mu \mathrm{l}$ of PBS. BAL was performed on a subset of mice at the indicated time by cannulation of the trachea and lavage with either $3 \mathrm{ml}$ (for cell assessment) or $1 \mathrm{ml}$ (for cytokine determination) of $\mathrm{PBS}^{28}$. Red blood cells were lysed and total nucleated cell counts were obtained using a haemocytometer. Cytospin slides were prepared by Wright-Giemsa staining with HEMA 3 (Fisher) and numbers of neutrophils, lymphocytes and dendritic cells/macrophages quantified. Alternatively, BAL cell differentials were determined by flow cytometry. At the indicated time, post infection blood was collected and blood count /differential was performed by the Clinical Pathology Laboratory at Iowa State University.

Microvascular permeability. We assessed microvascular permeability through extravasation of Evans blue dye. Thirty minutes before euthanasia, mice were injected intravenously with Evans blue dye $\left(20 \mathrm{mg} \mathrm{kg}^{-1}\right.$; Sigma). Lungs were perfused with PBS to remove intravascular Evans blue dye. Lungs were harvested and the Evans blue dye extracted as previously described ${ }^{29}$. Briefly, lungs were harvested, homogenized and incubated overnight with 16.7\% formamide in PBS at $37^{\circ} \mathrm{C}$. Lung homogenates were filtered through a $70 \mu \mathrm{m}$ mesh and plated into 96-well flat bottom plates. Absorbance at 620 and $740 \mathrm{~nm}$ were measured and used to correct for the presence of haeme pigments using the following equation: $A_{620}($ corrected $)=A_{620}-\left(1.426 \times A_{740}+0.03\right)($ refs 29,30$)$. The amount of extravasated Evans blue dye was then calculated against a standard curve.

Adoptive cell transfer. For adoptive transfer mice were injected intravenously in the tail vein with $5 \times 10^{6}$ bone marrow cells from WT or Nlrp12 $2^{-/-}$mice in PBS into congenically mismatched recipients before intranasal LPS challenge. Adoptive transfer of BMDM was accomplished by intranasal delivery of $4 \times 10^{6}$ BMDM derived from either donor WT or Nlrp12 ${ }^{-/-}$mice into the corresponding recipient; $16-18 \mathrm{~h}$ later mice were i.n. challenged with LPS.

Flow cytometry. Single cell suspensions of lungs prepared by pressing tissues through wire mesh screens or BAL were used and live cells enumerated using trypan blue exclusion. In all, $0.1-1 \times 10^{6}$ cells per well were stained in a 96-well plate and blocked with $2 \%$ rat serum or Fc Block (anti-mouse CD16/CD32; clone 2.4G2; 1:500; BioLegend) in FACS Buffer for $30 \mathrm{~min}$ at $4{ }^{\circ} \mathrm{C}$. Following blocking, BAL cells and lung homogenate cells were stained for $30 \mathrm{~min}$ at $4{ }^{\circ} \mathrm{C}$ with anti-CD45.2 (PerCP-Cy5.5 or PE-Cy7; clone 104; 1:100; BioLegend), anti-CD45.1 (FITC or PE; clone A20; 1:100; Biolegend), anti-CD11b (FITC, PE or PE-Cy7; clone M1/70; 1:400; eBioscience), anti-CD11c (PerCP-Cy5.5; clone N418; 1:200; eBioscience), anti-Gr1 (APC or FITC; clone RB6-8C5 or 1A8; 1:200; eBioscience) and anti-F4/80 (PE; clone BM8; 1:100; eBioscience) as described. Cells were subsequently fixed with FACS lysis buffer (BD Biosciences) per manufacturer's instructions and resuspended in PBS. For CXCL1 intracellular staining BMDMs were left unstimulated or stimulated for $4 \mathrm{~h}$ with LPS $\left(50 \mathrm{ng} \mathrm{ml}^{-1}\right)$ in the presence of brefeldin A (5 $\mathrm{g} \mathrm{ml}^{-1}$; BioLegend) and monensin $(2 \mu \mathrm{M}$; eBioscience). Cells were permeabilized and stained for the presence of intracellular CXCL1 using a biotinylated anti-mouse CXCL1 antibody (clone BAF453; 1:100; R\&D systems). Secondary staining was accomplished with streptavidin PE (eBioscience). Data were acquired on a BD LSR II and analysed using FlowJo Software (Treestar, Inc.).

In vitro stimulation of macrophages and dendritic cells. BMDM and BMDC $^{31,32}$ were either left unprimed or primed with $50 \mathrm{ng} \mathrm{ml}^{-1}$ LPS for $4 \mathrm{~h}$ and then infected with $F$. tularensis LVS (MOI 50:1), P. aeruginosa PAK strain (MOI 1:1), or S. aureus Newman strain (MOI 1:1) for $8 \mathrm{~h}$ and supernatants collected and assayed for cytokine levels by ELISA. For immunoblotting, cells were lysed in lysis buffer ( $50 \mathrm{mM}$ Tris $\cdot \mathrm{HCl}, 5 \mathrm{mM}$ EDTA, $150 \mathrm{mM} \mathrm{NaCl}, 1 \%$ Triton $\mathrm{X}-100$ and a protease inhibitor mixture (Roche)) and stored at $-80^{\circ} \mathrm{C}$ until 
analysed. Proteins were separated on a NuPAGE gel (Invitrogen) and transferred to a polyvinylidene difluoride membrane by electroblotting ${ }^{33}$. To detect caspase- 1 , rabbit polyclonal anti-mouse caspase-1 p10 antibody (sc-514; 1:100; Santa Cruz Biotechnology) was used. TLR agonists were obtained from InvivoGen and used to stimulate BMDM as follows: LPS, $50 \mathrm{ng} \mathrm{ml}^{-1}$; lipoteichoic acid (LTA), $5 \mu \mathrm{g} \mathrm{ml}^{-1}$; FSL1 (Pam2CGDPKHPKSF), $1 \mu \mathrm{g} \mathrm{ml}^{-1}$; heat-killed Listeria monocytogenes (HKLM), $1 \times 10^{8}$ cells ml $^{-1}$; Pam3csk, $1 \mu \mathrm{g} \mathrm{ml}^{-1}$; and R848, $4 \mu \mathrm{g} \mathrm{ml}^{-1}$. Cells were stimulated for $8 \mathrm{~h}$ or the indicated time and supernatants collected and assayed for cytokine levels by ELISA. Cell lysates were used for immunoblotting; the following antibodies from Cell Signaling Technology were utilized at 1:1,000: phospho-p38 MAPK (Thr180/Tyr182, product \#4511, clone D3F9); total p38 MAPK (product \#8690, clone D13E1); phospho-p44/42 MAPK (Thr202/Tyr204, product \#4370, clone D13.14.4E); total p44/42 MAPK (product \#4695, clone 137F5), phospho-SAPK/JNK (Thr183/Tyr185, product \#4668, clone 81E11); total SAPK/JNK (product \#9252, polyclonal); phospho-IкB $\alpha$ (Ser32, product \#2859,

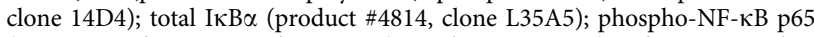
(Ser536, product \#3033, clone $93 \mathrm{H1}$ ); total NF- $\kappa \mathrm{B}$ p65 (product \#8242, clone D14E12); NF- $\kappa$ B2 p100/p52 (product \#4882, clone 18D10); and $\alpha$-tubulin (product \#2125, clone $11 \mathrm{H} 10$ ).

Cytokine ELISA. Culture supernatants and BAL samples were assayed for IL-1 $\beta$, MIP- $1 \alpha$, TNF $\alpha$, IL-6, IL-10, IL-18, IL-17A and CXCL1 by ELISA. Antibody pairs for the IL-1 $\beta$ (MAB401 and BAF401; clone 30311 and polyclonal; R\&D Systems; 8 and $4 \mu \mathrm{g} \mathrm{ml}^{-1}$, respectively) and CXCL1 (mouse: MAB453 and BAF453; clone 48415 and polyclonal; R\&D Systems; 8 and $0.4 \mu \mathrm{g} \mathrm{ml}^{-1}$, respectively; human: MAB275, clone 20326, $4 \mathrm{\mu g} \mathrm{ml}^{-1}$; BAF275, polyclonal, $40 \mathrm{ng} \mathrm{ml}^{-1}$ ) and CCL3/ MIP- $1 \alpha$ (Quantikine ELISA kit, Product \#MMA00) ELISAs were from R\&D Systems. Antibody pairs for IL-1 $\alpha$ (capture: product \#14-7011-85, clone ALF-161; detection: product \#13-7111-85, polyclonal), TNF $\alpha$ (capture: product \#14-7325-85, clone 1F3F3D4; detection: product \#13-7326-85, clones MP6-XT22 and MP6-XT3), IL-6 (capture: product \#14-7061-85, clone MP5-20F3; detection: product \#13-7062-85, clone MP5-32C11), IL-10 (capture: product \#14-7101-85, clone JES5-16E3; detection: product \#13-7102-85, clone JES5-2A5) and IL-17A (capture: product \#14-7175-85, clone eBio 17CK15A5; detection: product \#13-7177-85, clone eBio17B7) were from eBiosciences and used at a 1:250 dilution. Antibody pairs for IL-18 were from MBL International (capture: product \#D047-3, clone 74, 1:1,000; detection: product \#D048-6, clone 93-10C, 1:2,000).

Transfection. To prepare vectors with $\mathrm{B} 6 \mathrm{~N}$ and $\mathrm{B} 6 \mathrm{~J}$ Nlrp12 variants we mutated $\mathrm{Nlrp} 12^{B 6 J}$ in the pCMV6-AC-GFP vector (Origene Technologies) to $N \operatorname{lrp} 12^{B 6 N}$ using the Quickchange II XL Site-Directed Mutagenesis Kit (Agilent Technologies) according to the manufacturer's instructions and the duplex primers $5^{\prime}$-GCTGT TTGGGATGGACCTGAATAGAAAGACTCACAGGAGGATGGC- ${ }^{\prime}{ }^{\prime}$ with the reverse complement (Integrated DNA Technologies). The sequence of all $N \operatorname{lrp} 12^{B 6 N_{-}}$and Nlrp12B6J-containing vectors were validated by Sanger sequencing (Iowa Institute of Human Genetics, Genomics Division). TLR4-expressing HEK293 cells (HEK-TLR4) (ref. 34) were a generous gift from Dr Jerrold Weiss at the University of Iowa. HEK-TLR4 cells were transfected with $2 \mu \mathrm{g}$ of the GFP-tagged $N \operatorname{lrp} 12^{B 6 \mathrm{~N}}$ or Nlrp12 ${ }^{B 6 J}$ constructs using Lipofectamine 3000 (Thermo Fisher Scientific) according to the manufacturer's instructions. Twenty-four hours after transfection, cells were stimulated for $16 \mathrm{~h}$ with $100 \mathrm{ng} \mathrm{ml}^{-1}$ LPS.

Supernatants were used for ELISA and cell lysates were used for immunoblotting. Anti-turboGFP (OTI2H8) from Origene Technologies was used to detect the GFP tag in transfected cells.

Real-time PCR and sequencing. Complementary DNA was generated at the indicated time post stimulation with LPS and real-time PCR performed using PerfeCTa SYBR Green Fast Mix (Quanta) and RT2 quantitative PCR primers (Qiagen) for Cxcl1. For quantification, standards were generated by cloning the PCR product into the pCR 2.1 vector using TOPO TA cloning kit (Life Technologies).

To identify genetic differences between C57BL/6N and C57BL/6J mice the SNP for Nlrp12 and indels for $\mathrm{Crb} 1$ and $\mathrm{N} n t$ genes were PCR amplified using the following primers. Nlrp12: forward primer (5'-CTGGGAGCTGGATTT- $\left.3^{\prime}\right)$, reverse primer ( $5^{\prime}$-TATCCTGGTCGGCTTCATTC- $\left.3^{\prime}\right)$; Crb1: forward primer ( $5^{\prime}$ - CGAGTGGACT TTGTGCTCACC- $\left.3^{\prime}\right)$, reverse primer $\left(5^{\prime}\right.$ - TTGACAATCCGAAAGGCCTGC- $\left.3^{\prime}\right)$; Nnt common primer ( $5^{\prime}$-GTAGGGCCAACTGTTTCTGCATGA-3'), WT primer ( $5^{\prime}$-GGGCATAGGAAGCAAATACCAAGTTG- $\left.{ }^{\prime}\right)$ and mutant primer $\left(5^{\prime}\right.$-GTGGA ATTCCGCTGAGAGAACTCTT- $\left.3^{\prime}\right)^{35}$. Once amplified SNP and indel differences were either identified by Sanger sequencing (Nlrp12 and Crb1) or by running PCR products on a $1 \%$ agarose gel $(\mathrm{Nnt})$.

Data availability. The data that support the findings of this study are available from the corresponding author upon request.

\section{References}

1. Simon, M. M. et al. A comparative phenotypic and genomic analysis of C57BL/6J and C57BL/6N mouse strains. Genome Biol. 14, R82 (2013).

2. Vanden Berghe, T. et al. Passenger mutations confound interpretation of all genetically modified congenic mice. Immunity 43, 200-209 (2015).
3. Mekada, K. et al. Genetic differences among C57BL/6 substrains. Exp. Anim. 58, 141-149 (2009).

4. Schroder, K. \& Tschopp, J. The inflammasomes. Cell 140, 821-832 (2010).

5. Wang, L. et al. PYPAF7, a novel PYRIN-containing Apaf1-like protein that regulates activation of NF-kappa B and caspase-1-dependent cytokine processing. J. Biol. Chem. 277, 29874-29880 (2002).

6. Williams, K. L. et al. The CATERPILLER protein monarch-1 is an antagonist of toll-like receptor-, tumor necrosis factor alpha-, and Mycobacterium tuberculosis-induced pro-inflammatory signals. J. Biol. Chem. 280, 39914-39924 (2005).

7. Lich, J. D. et al. Monarch-1 suppresses non-canonical NF-kappaB activation and p52-dependent chemokine expression in monocytes. J. Immunol. 178, 1256-1260 (2007).

8. Allen, I. C. et al. NLRP12 suppresses colon inflammation and tumorigenesis through the negative regulation of noncanonical NF-kappaB signaling. Immunity 36, 742-754 (2012).

9. Zaki, M. H. et al. The NOD-like receptor NLRP12 attenuates colon inflammation and tumorigenesis. Cancer Cell 20, 649-660 (2011).

10. Vladimer, G. I. et al. The NLRP12 inflammasome recognizes Yersinia pestis. Immunity 37, 96-107 (2012).

11. Zaki, M. H., Man, S. M., Vogel, P., Lamkanfi, M. \& Kanneganti, T. D. Salmonella exploits NLRP12-dependent innate immune signaling to suppress host defenses during infection. Proc. Natl Acad. Sci. USA 111, 385-390 (2013).

12. Allen, I. C. et al. Characterization of NLRP12 during the in vivo host immune response to Klebsiella pneumoniae and Mycobacterium tuberculosis. PLoS ONE 8, e60842 (2013).

13. Bourdi, M., Davies, J. S. \& Pohl, L. R. Mispairing C57BL/6 substrains of genetically engineered mice and wild-type controls can lead to confounding results as it did in studies of JNK2 in acetaminophen and concanavalin A liver injury. Chem. Res. Toxicol. 24, 794-796 (2011).

14. Ivanov, I. I. et al. Induction of intestinal Th17 cells by segmented filamentous bacteria. Cell 139, 485-498 (2009).

15. Zhang, Y. M. F(2) designs for QTL analysis. Methods Mol. Biol. 871, 17-29 (2012).

16. Kumar, V. et al. C57BL/6N mutation in cytoplasmic FMRP interacting protein 2 regulates cocaine response. Science 342, 1508-1512 (2013).

17. Mariathasan, S., Weiss, D. S., Dixit, V. M. \& Monack, D. M. Innate immunity against Francisella tularensis is dependent on the ASC/caspase-1 axis. J. Exp. Med. 202, 1043-1049 (2005).

18. Ulland, T. K. et al. Francisella tularensis live vaccine strain folate metabolism and pseudouridine synthase gene mutants modulate macrophage caspase-1 activation. Infect. Immun. 81, 201-208 (2013).

19. Kolaczkowska, E. \& Kubes, P. Neutrophil recruitment and function in health and inflammation. Nat. Rev. Immunol. 13, 159-175 (2013).

20. Cai, S., Batra, S., Del Piero, F. \& Jeyaseelan, S. NLRP12 modulates host defense through IL-17A-CXCL1 axis. Mucosal Immunol. 9, 503-514 (2016).

21. Arthur, J. C. et al. Cutting edge: NLRP12 controls dendritic and myeloid cell migration to affect contact hypersensitivity. J. Immunol. 185, 4515-4519 (2010).

22. Zamoshnikova, A. et al. NLRP12 is a neutrophil-specific, negative regulator of in vitro cell migration but does not modulate LPS- or infection-induced NF-kappaB or ERK signalling. Immunobiology 221, 341-346 (2016).

23. Abtin, A. et al. Perivascular macrophages mediate neutrophil recruitment during bacterial skin infection. Nat. Immunol. 15, 45-53 (2014).

24. Elinav, E. et al. NLRP6 inflammasome regulates colonic microbial ecology and risk for colitis. Cell 145, 745-757 (2011).

25. Sutterwala, F. S. et al. Critical role for NALP3/CIAS1/Cryopyrin in innate and adaptive immunity through its regulation of caspase-1. Immunity 24, 317-327 (2006).

26. Eisenbarth, S. C. et al. NLRP10 is a NOD-like receptor essential to initiate adaptive immunity by dendritic cells. Nature 484, 510-513 (2012).

27. Schwartz, J. T. et al. Natural IgM mediates complement-dependent uptake of Francisella tularensis by human neutrophils via complement receptors 1 and 3 in nonimmune serum. J. Immunol. 189, 3064-3077 (2012).

28. Cohn, L., Homer, R. J., Niu, N. \& Bottomly, K. T helper 1 cells and interferon gamma regulate allergic airway inflammation and mucus production. J. Exp. Med. 190, 1309-1318 (1999).

29. Green, T. P., Johnson, D. E., Marchessault, R. P. \& Gatto, C. W. Transvascular flux and tissue accrual of Evans blue: effects of endotoxin and histamine. J. Lab. Clin. Med. 111, 173-183 (1988).

30. Reutershan, J. et al. Critical role of endothelial CXCR2 in LPS-induced neutrophil migration into the lung. J. Clin. Invest. 116, 695-702 (2006).

31. Sutterwala, F. S., Noel, G. J., Clynes, R. \& Mosser, D. M. Selective suppression of interleukin-12 induction after macrophage receptor ligation. J. Exp. Med. 185, 1977-1985 (1997).

32. Goodridge, H. S. et al. Differential use of CARD9 by dectin-1 in macrophages and dendritic cells. J. Immunol. 182, 1146-1154 (2009).

33. Cassel, S. L. et al. The Nalp3 inflammasome is essential for the development of silicosis. Proc. Natl Acad. Sci. USA 105, 9035-9040 (2008). 
34. Yang, H., Young, D. W., Gusovsky, F. \& Chow, J. C. Cellular events mediated by lipopolysaccharide-stimulated toll-like receptor 4 . MD-2 is required for activation of mitogen-activated protein kinases and Elk-1. J. Biol. Chem. 275 20861-20866 (2000).

35. Navarro, S. J. et al. The C57BL/6J mouse strain background modifies the effect of a mutation in Bcl2l2. G3 (Bethesda) 2, 99-102 (2012).

\section{Acknowledgements}

We thank Richard Flavell and Millennium Pharmaceuticals for providing knockout mice, William Nauseef for helpful discussion and Vickie Knepper-Adrian for technical assistance. NIH grants R01 AI118719 (F.S.S.), P01 AI44642 (F.S.S.), the Midwest Regional Center of Excellence project grant U54 AI057160 (F.S.S.), R01 AI104706 (S.L.C.), R01 AR059703 (P.J.F.), T32 AI007485 (A.M.J.) and an Asthma and Allergy Foundation of America fellowship (S.L.C.) supported this work.

\section{Author contributions}

Conceptualization: T.K.U., N.J., S.L.C. and F.S.S.; methodology: T.K.U., N.J., S.L.C. and F.S.S.; investigation: T.K.U., N.J., G.C.C., E.E.H., E.I.E., J.J.S., K.A.M.M., and A.M.J.; formal analysis: K.W. and L.G.; resources: K.L.L.; writing original draft: T.K.U., S.L.C. and F.S.S.; writing review and editing: T.K.U., N.J., E.E.H., A.P.D.V., K.L.L., M.B., P.J.F., M.E.W., S.L.C. and F.S.S; funding acquisition: S.L.C. and F.S.S., supervision: S.L.C. and F.S.S.

\section{Additional information}

Supplementary Information accompanies this paper at http://www.nature.com/ naturecommunications

Competing financial interests: The authors declare no competing financial interests.

Reprints and permission information is available online at http://npg.nature.com/ reprintsandpermissions/

How to cite this article: Ulland, T. K. et al. Nlrp12 mutation causes C57BL/6J strain-specific defect in neutrophil recruitment. Nat. Commun. 7, 13180 doi: $10.1038 /$ ncomms13180 (2016).

\section{(c) (i)}

This work is licensed under a Creative Commons Attribution 4.0 International License. The images or other third party material in this article are included in the article's Creative Commons license, unless indicated otherwise in the credit line; if the material is not included under the Creative Commons license, users will need to obtain permission from the license holder to reproduce the material. To view a copy of this license, visit http://creativecommons.org/licenses/by/4.0/

(C) The Author(s) 2016 